ALEA, Lat. Am. J. Probab. Math. Stat. 14, 403-426 (2017)

DOI: 10.30757/ALEA.v14-22

\title{
Limit theorems for the sample mean and sample autocovariances of continuous time moving averages driven by heavy-tailed Lévy noise
}

\author{
Martin Drapatz \\ Ulm University \\ Institute for Mathematical Finance \\ Helmholtzstraße 18 \\ 89081 Ulm, Germany. \\ E-mail address: martin.drapatz@uni-ulm.de
}

\begin{abstract}
In this article we consider continuous time moving averages observed on a lattice, driven by an infinite variance Lévy process with regularly varying tails with index $\alpha \in(0,2)$. We show that the asymptotic distribution of the sample mean and sample autocovariance function is a stable law. The parameters of the stable limit distribution are explicitly given in terms of the kernel and characteristics of the Lévy process.
\end{abstract}

\section{Introduction}

Continuous time moving averages appear in many fields of applied probability as economics and finance and cover many popular models from time series analysis in continuous time. Because of their applicability to irregularly spaced observations and high-frequency data, frequently appearing in finance and turbulence, continuous time moving averages are often favored over their discrete time counterparts. Let $L=\left(L_{t}\right)_{t \in \mathbb{R}}$ be a two-sided one-dimensional Lévy process, i.e. a stochastic process with $L_{0}=0$, independent and stationary increments and càdlàg paths. In this paper we will consider continuous time moving averages of type

$$
X_{t}=\int_{\mathbb{R}} f(t-s) \mathrm{d} L_{s}, \quad t \in \mathbb{R}
$$

where $f: \mathbb{R} \rightarrow \mathbb{R}$ is a measurable function and the Lévy process $L=\left(L_{t}\right)_{t \in \mathbb{R}}$ has regularly varying tails with index $\alpha \in(0,2)$ and in particular infinite variance. Many popular models in continuous time as e.g. CARMA processes and OrnsteinUhlenbeck processes can be represented by continuous time moving averages and

Received by the editors March 16th, 2016; accepted February 24th, 2017.

2010 Mathematics Subject Classification. 60F05, 60G10.

Key words and phrases. continuous time moving average, Lévy process, sample autocovariance, sample mean. 
methods for the estimation of the kernel function $f$ are available (see e.g. Brockwell et al., 2013 and Fasen, 2013, and for the special case of a stable driving Lévy process Fasen and Fuchs, 2013 and García et al., 2011). Our purpose will be to determine the asymptotic distribution of the sample mean and sample autocovariances of $\left(X_{t}\right)_{t \in \mathbb{R}}$ in (1.1), observed on a lattice $\{\Delta t: t=1,2, \ldots\}$ for some $\Delta>0$.

The asymptotic distribution of the mean and autocovariance function of continuous time moving averages has been already investigated by many others. Cohen and Lindner (2013) proved a central limit theorem for the mean and autocovariance function under the assumptions $\mathbb{E} L_{1}^{2}<\infty$ (respectively $\mathbb{E} L_{1}^{4}<\infty$ for the autocovariances) and square-integrable kernel $f$. Spangenberg (2015) studied the long memory case where $f(t) \sim C_{d} t^{d-1}$ for $d \in\left(0, \frac{1}{2}\right)$ and some constant $C_{d}$. When $\mathbb{E} L_{1}^{4}<\infty$ he proved a central limit theorem and in the case of a Lévy process with regularly varying tails with index $\alpha \in(2,4)$ that the limit distribution is either a Rosenblatt or stable distribution, depending on the interplay of $d$ and $\alpha$. Further, Bai et al. (2016) considered certain Toeplitz type quadratic functionals of continuous time moving averages with square integrable kernel $f$ and finite variance Lévy processes which arise in the statistical estimation of the spectrum of stationary processes.

For discrete time moving averages of the form $X_{t}=\sum_{k=0}^{\infty} c_{k} Z_{t-k}$ for $\left(c_{k}\right)_{k \in \mathbb{N}_{0}} \subset$ $\mathbb{R}$ and $t \in \mathbb{Z}$ where $\left(Z_{t}\right)_{t \in \mathbb{Z}}$ is an i.i.d. sequence of random variables which are regularly varying with index $\alpha \in(0,2)$ and satisfy a tail balance condition, Davis and Resnick (1985) established that the sample mean and the sample autocovariances are asymptotic stable distributed. Their approach was based on point process methods. We will use a different approach which is based on classical blocking and mixing techniques, and is due to Jakubowski (1993, 1997), see also Bartkiewicz et al. (2011) for a survey on stable limit theorems under dependence. The main advantage compared to the point process approach is the more explicit representation of the parameters of the stable distribution.

We will use the following notations: the indicator function of a set $A$ is denoted by $\mathbb{1}_{A}$ and the signum function $\operatorname{sign}(z)$ is defined by $\operatorname{sign}(z)=\mathbb{1}(z>0)-\mathbb{1}(z<0)$. The boundary of a set $A$ is denoted by $\partial A$. By $\mathcal{B}(A)$ for a set $A$ we denote the Borel$\sigma$-algebra on $A$. The symbols $\stackrel{d}{\rightarrow}, \stackrel{w}{\rightarrow}, \stackrel{v}{\rightarrow}$ and $\stackrel{\mathbb{P}}{\rightarrow}$ denote convergence in distribution, weak convergence, vague convergence and convergence in probability. Sometimes we write for a measure $\nu$ and a set $A$ short $\nu A$ for $\nu(A)$. By $f^{+}$and $f^{-}$we denote the positive and negative part of a function $f$.

\section{Preliminaries}

In this article we assume that $\left(L_{t}\right)_{t \in \mathbb{R}}$ is a two-sided one-dimensional Lévy process with triplet $\left(\gamma, \sigma^{2}, \nu\right)$, i.e. the characteristic function of $L_{t}$ is given by $\varphi_{L_{t}}(z)=\mathbb{E} \mathrm{e}^{i z L_{t}}=\mathrm{e}^{|t| \psi(z \operatorname{sign}(t))}$ where the characteristic exponent $\psi(z)$ admits the representation (see Sato, 1999, Theorem 8.1)

$$
\psi(z)=\mathrm{i} \gamma z-\frac{\sigma^{2} z^{2}}{2}+\int_{\mathbb{R}}\left[\mathrm{e}^{\mathrm{i} z x}-1-\mathrm{i} z x \mathbb{1}(|x| \leq 1)\right] \nu(d x), \quad z \in \mathbb{R} .
$$

Here $\gamma \in \mathbb{R}$ is called generalized drift, $\sigma^{2} \geq 0$ the Gaussian part and $\nu$ is the Lévy measure, a measure on $\left(\mathbb{R}, \mathcal{B}_{1}\right)$ such that $\nu(\{0\})=0$ and $\int_{\mathbb{R} \backslash\{0\}}\left(|x|^{2} \wedge 1\right) \nu(d x)<\infty$. Denote by $\bar{\nu}(x)=\nu(x, \infty)+\nu(-\infty,-x)$ for $x>1$ the Lévy tail measure. 
We will use the notion of multivariate regular variation (see Hult and Lindskog, 2006). Set $\overline{\mathbb{R}}^{d}=[-\infty, \infty]^{d}$. An $\mathbb{R}^{d}$-valued random vector $X=\left(X_{1}, \ldots, X_{d}\right)^{T}$ with unbounded support is multivariate regularly varying, if there are an $\alpha>0$, a positive sequence $\left(a_{n}\right)_{n \in \mathbb{N}}, a_{n} \uparrow \infty$, and a nonzero Radon measure $\mu$ defined on $\mathcal{B}\left(\overline{\mathbb{R}}^{d} \backslash\{0\}\right), \mu\left(\overline{\mathbb{R}}^{d} \backslash \mathbb{R}^{d}\right)=0$, such that, as $n \rightarrow \infty$,

$$
n \mathbb{P}\left(X \in a_{n} \cdot\right) \stackrel{v}{\rightarrow} \mu(\cdot) \quad \text { on } \mathcal{B}\left(\overline{\mathbb{R}}^{d} \backslash\{0\}\right) .
$$

We write $X \in R V\left(\alpha, a_{n}, \mu\right)$. Similarly, we write for a Radon measure $\nu$ on $\mathcal{B}\left(\overline{\mathbb{R}}^{d} \backslash\{0\}\right), \nu \in R V\left(\alpha, a_{n}, \mu\right)$, if $n \nu\left(a_{n} \cdot\right) \stackrel{v}{\rightarrow} \mu(\cdot)$ on $\mathcal{B}\left(\overline{\mathbb{R}}^{d} \backslash\{0\}\right)$. Notice that the limiting measure has the property $\mu(x A) \sim x^{-\alpha} \mu(A)$ for $x>0$ and every set $A \in \mathcal{B}\left(\overline{\mathbb{R}}^{d} \backslash\{0\}\right)$, see e.g. Hult and Lindskog (2006). If $d=1$ and the Lévy process $\left(L_{t}\right)_{t \in \mathbb{R}}$ satisfies $L_{1} \in R V\left(\alpha, a_{n}, \mu\right)$ for a sequence $\left(a_{n}\right)_{n \in \mathbb{N}}$ with

$$
n \mathbb{P}\left(\left|L_{1}\right| \geq a_{n}\right) \rightarrow 1, \quad n \rightarrow \infty,
$$

then the limiting measure $\mu$ is explicitly given by

$$
\mu(\mathrm{d} x)=\left(p \mathbb{1}_{(0, \infty)}(x)+q \mathbb{1}_{(-\infty, 0)}(x)\right) \alpha|x|^{-\alpha-1} \mathrm{~d} x,
$$

where the constants $p$ and $q$ are explicitly given by

$$
\lim _{x \rightarrow \infty} \frac{\mathbb{P}\left(L_{1}>x\right)}{\mathbb{P}\left(\left|L_{1}\right|>x\right)}=p, \quad \lim _{x \rightarrow \infty} \frac{\mathbb{P}\left(L_{1}<-x\right)}{\mathbb{P}\left(\left|L_{1}\right|>x\right)}=q, \quad p+q=1, \quad p, q \in[0,1] .
$$

If $L_{1} \in R V\left(\alpha, b_{n}, \widetilde{\mu}\right)$ for some $\left(b_{n}\right)_{n \in \mathbb{N}}$ and $\widetilde{\mu}$, and we set $a_{n}=\inf \left(x: \mathbb{P}\left(\left|L_{1}\right|>\right.\right.$ $\left.x) \leq \frac{1}{n}\right)$ for $n \in \mathbb{N}$, then $\left(a_{n}\right)_{n \in \mathbb{N}}$ satisfies $(2.1)$ and $L_{1} \in R V\left(\alpha, a_{n}, \mu\right)$ for some $\mu$. In this case the sequence $\left(a_{n}\right)_{n \in \mathbb{N}}$ is regularly varying with index $\alpha^{-1}$ and behaves therefore like

$$
a_{n} \sim L(n) n^{\alpha^{-1}}, \quad n \rightarrow \infty
$$

for some slowly varying function $L$ and $n \in \mathbb{N}$.

Remark 1. If $X$ is an infinitely divisible random variable with triplet $\left(\gamma, \sigma^{2}, \nu\right)$, then $X \in R V\left(\alpha, a_{n}, \mu\right)$ if and only if $\nu \in R V\left(\alpha, a_{n}, \mu\right)$, see Pakes (2007, Theorem 3.3) and also Embrechts et al. (1979) for one-sided distributions. Hult and Lindskog (2006, Proposition 3.1, Corollary 3.1) proved the analogue statement for multivariate infinitely divisible distributions and the notion of multivariate regular variation. We will use above tail equivalence only for $d=1$, but note that it is implicitly used for $d>1$ in the proof of Proposition 1 (i) (see Moser and Stelzer, 2011, Theorem 3.1). Note further that there are many equivalent definitions of multivariate regular variation, see e.g. Lindskog et al. (2014, Theorem 3.1). Equation (2.3) is often referred to as tail balance condition. In particular, if $p>0$ and $q>0$, by Hult and Lindskog (2006, Corollary 3.1) as $x \rightarrow \infty$

$$
\mathbb{P}\left(L_{1}>x\right) \sim \nu(x, \infty) \sim \nu[x, \infty), \quad \mathbb{P}\left(L_{1}<-x\right) \sim \nu(-\infty,-x) \sim \nu(-\infty,-x] .
$$

Also note that $\mathbb{P}\left(L_{1}>x\right) \sim \nu[x, \infty)$ entails $\mathbb{P}\left(L_{t}>x\right) \sim t \nu[x, \infty)$ for $t>0$ and $x \rightarrow \infty$.

In the following we will consider continuous time moving averages of the form

$$
X_{t}=\int_{\mathbb{R}} f(t-s) \mathrm{d} L_{s}, \quad t \in \mathbb{R},
$$


where $f: \mathbb{R} \rightarrow \mathbb{R}$ is a measurable function and $L=\left(L_{t}\right)_{t \in \mathbb{R}}$ is a one-dimensional Lévy process with triplet $\left(\gamma, \sigma^{2}, \nu\right)$. The stochastic integral (2.5) is defined as follows: let $a<b, a, b \in \mathbb{R}$, and $f:(a, b) \rightarrow \mathbb{R}$ be a measurable function. Then $f$ is called $L$-integrable, if for every sequence of simple functions $\left(f_{n}\right)_{n \in \mathbb{N}}$ such that $f_{n} \rightarrow f$ almost everywhere, the limit $\mathbb{P}-\lim _{n \rightarrow \infty} \int_{(a, b)} f_{n}(s) \mathrm{d} L_{s}$ exists. In this case we set $\int_{(a, b)} f(s) \mathrm{d} L_{s}:=\mathbb{P}-\lim _{n \rightarrow \infty} \int_{(a, b)} f_{n}(s) \mathrm{d} L_{s}$ and the limit does not depend on the choice of the sequence of simple functions. If further for a function $f: \mathbb{R} \rightarrow \mathbb{R}$ the limit $\mathbb{P}-\lim _{p, q \rightarrow \infty} \int_{-p}^{q} f(s) \mathrm{d} L_{s}$ exists, the stochastic integral $\int_{-\infty}^{\infty} f(s) \mathrm{d} L_{s}$ is called defineable, the function $f$ is called (improper) $L$-integrable, and $X_{t}$ in (2.5) is set equal to $\mathbb{P}-\lim _{p, q \rightarrow \infty} \int_{-p}^{q} f(t-s) \mathrm{d} L_{s}$, in which case the distribution of $X_{t}$ is infinitely divisible. Since the Lévy process has the independent increment property, the convergence is also almost surely. Necessary and sufficient conditions in terms of $f$ and the characteristic triplet $\left(\gamma, \sigma^{2}, \nu\right)$ for the stochastic integral (2.5) being definable are well-known (see Sato, 2006 or Rajput and Rosiński, 1989).

Denote by $L^{p}(A)$ for $A \in \mathcal{B}(\mathbb{R})$ and $0<p<\infty$ the set of functions $f: A \rightarrow \mathbb{R}$ which satisfy $\int_{A}|f(s)|^{p} \mathrm{~d} s<\infty$. Throughout this article we will assume that the following two conditions are satisfied.

Condition (A1). The Lévy process $\left(L_{t}\right)_{t \in \mathbb{R}}$ satisfies $L_{1} \in R V\left(\alpha, a_{n}, \mu\right)$ for some $\alpha \in(0,2)$, a positive sequence $\left(a_{n}\right)_{n \in \mathbb{N}}, a_{n} \uparrow \infty$, satisfying $(2.1)$ and $\mu(\mathrm{d} x)=$ $\left(p \mathbb{1}_{(0, \infty)}(x)+q \mathbb{1}_{(-\infty, 0)}(x)\right) \alpha|x|^{-\alpha-1} \mathrm{~d} x$, where the constants $p$ and $q$ are given by

$$
\lim _{x \rightarrow \infty} \frac{\mathbb{P}\left(L_{1}>x\right)}{\mathbb{P}\left(\left|L_{1}\right|>x\right)}=p, \quad \lim _{x \rightarrow \infty} \frac{\mathbb{P}\left(L_{1}<-x\right)}{\mathbb{P}\left(\left|L_{1}\right|>x\right)}=q, \quad p+q=1, \quad p, q \in[0,1] .
$$

Further, assume that for $\alpha>1$ the expectation of $L_{1}$ is zero, i.e. $\mathbb{E} L_{1}=0$.

Condition (A2). The kernel $f: \mathbb{R} \rightarrow \mathbb{R}$ is bounded, $f \in L^{\delta}(\mathbb{R})$ for some $\delta<\alpha, \delta \leq 1$ and $\int_{\mathbb{R}}|f(s)| \mathrm{d} s \neq 0$.

Under above conditions (A1) and (A2) the stochastic integral (2.5) is definable, see Fasen (2005, Proposition 3.1). Observe that $f \in L^{\delta}(\mathbb{R})$ implies $f \in L^{\delta+\epsilon}(\mathbb{R})$ for bounded $f: \mathbb{R} \rightarrow \mathbb{R}$ and $\delta, \epsilon>0$.

\section{Results}

In this section we state our main results. We establish limit theorems for the sample mean and sample autocovariances of continuous time moving averages of type (2.5). In both cases the limit distribution will be a stable law. Two random variables $X$ and $Y$, and also their distributions, are said to belong to the same type,

if they have the same distribution after change of scale and location, i.e. $X \stackrel{d}{=} a Y+b$ for some $a>0$ and $b \in \mathbb{R}$. Stable distributions are distributions which are closed under convolutions. More precisely, a distribution $F$ is stable if for i.i.d. random variables $X$ and $Y, X, Y \sim F$, the distribution of the sum $X+Y$ is of same type as $F$. The characteristic function $\varphi_{X}$ of a (nondeterministic) stable random variable $X$ admits for $z \in \mathbb{R}$ the unique representation (see Nolan, 2017, Proposition 1.17)

$$
\begin{aligned}
\varphi_{X}(z) & =\exp \left(\mathrm{i} z \delta-|\gamma z|^{\alpha}\left(1-\mathrm{i} \beta \operatorname{sign}(z) \Phi_{\alpha}(z)\right)\right), \\
\text { where } \Phi_{\alpha}(z) & = \begin{cases}\tan \left(\frac{\pi \alpha}{2}\right), & \alpha \neq 1, \\
-\frac{2}{\pi} \log |z|, & \alpha=1,\end{cases}
\end{aligned}
$$


and $\alpha \in(0,2], \beta \in[-1,1], \gamma>0, \delta \in \mathbb{R}$. We write $X \sim S_{\alpha}(\beta, \gamma, \delta)$. Notice that for $X \sim S_{\alpha}(\beta, \gamma, \delta)$ and $a, b \in \mathbb{R}, a \neq 0$ (see also Nolan, 2017, Proposition 1.17)

$$
a X+b \sim \begin{cases}S_{\alpha}(\operatorname{sign}(a) \beta,|a| \gamma, a \delta+b), & \alpha \neq 1, \\ S_{\alpha}\left(\operatorname{sign}(a) \beta,|a| \gamma, a \delta+b-\frac{2}{\pi} \beta a \log (|a|)\right), & \alpha=1 .\end{cases}
$$

Define for $\Delta>0$

$$
\begin{array}{cc}
F_{\Delta}:[0, \Delta] \rightarrow \mathbb{R}, \quad u \mapsto \sum_{j=-\infty}^{\infty} f(\Delta j-u), \\
\bar{F}_{\Delta}:[0, \Delta] \rightarrow[0, \infty], \quad u \mapsto \sum_{j=-\infty}^{\infty}|f(\Delta j-u)| .
\end{array}
$$

Observe that (A2) ensures $\bar{F}_{\Delta}<\infty$ almost everywhere and $F_{\Delta} \in L^{1}([0, \Delta])$. We can now state our first main result:

Theorem 1. Let $\Delta>0$, conditions (A1) and (A2) be satisfied and set $X_{t}=$ $\int_{-\infty}^{\infty} f(t-s) \mathrm{d} L_{s}$. Suppose further that the distribution of $L_{1}$ is symmetric if $\alpha=1$ and that $\bar{F}_{\Delta} \in L^{2}([0, \Delta])$. Define the coefficients $c_{\Delta}^{+}$and $c_{\Delta}^{-}$by

$$
c_{\Delta}^{+}=\int_{0}^{\Delta}\left(p\left(F_{\Delta}^{+}(s)\right)^{\alpha}+q\left(F_{\Delta}^{-}(s)\right)^{\alpha}\right) \mathrm{d} s, \quad c_{\Delta}^{-}=\int_{0}^{\Delta}\left(q\left(F_{\Delta}^{+}(s)\right)^{\alpha}+p\left(F_{\Delta}^{-}(s)\right)^{\alpha}\right) \mathrm{d} s .
$$

(i) If $c_{\Delta}^{+}+c_{\Delta}^{-}>0$, then $a_{n}^{-1} \sum_{t=1}^{n} X_{\Delta t} \stackrel{d}{\rightarrow} S_{\alpha}\left(\beta_{\Delta}, \gamma_{\Delta}, 0\right)$ as $n \rightarrow \infty$, where the parameters are given by

$$
\begin{aligned}
& \beta_{\Delta}=(p-q) \frac{\int_{0}^{\Delta}\left(F_{\Delta}^{+}(s)\right)^{\alpha}-\left(F_{\Delta}^{-}(s)\right)^{\alpha} \mathrm{d} s}{\int_{0}^{\Delta}\left|F_{\Delta}(s)\right|^{\alpha} \mathrm{d} s}, \\
& \gamma_{\Delta}^{\alpha}= \begin{cases}\left(c_{\Delta}^{+}+c_{\Delta}^{-}\right) \frac{\Gamma(2-\alpha)}{(1-\alpha)} \cos \left(\frac{\pi \alpha}{2}\right), & \alpha \neq 1, \\
\left(c_{\Delta}^{+}+c_{\Delta}^{-}\right) \frac{\pi}{2}, & \alpha=1 .\end{cases}
\end{aligned}
$$

(ii) If $c_{\Delta}^{+}+c_{\Delta}^{-}=0$, then $a_{n}^{-1} \sum_{t=1}^{n} X_{\Delta t} \stackrel{\mathbb{P}}{\rightarrow} 0$ as $n \rightarrow \infty$.

Remark 2. (i) Notice that $f \in L^{1}(\mathbb{R})$ is necessary for $\bar{F}_{\Delta} \in L^{2}([0, \Delta])$ (see Cohen and Lindner, 2013, Remark 2.3). In the discrete time case (i.e. when $f=$ $\sum_{j \in \mathbb{Z}} c_{j} \mathbb{1}_{(\Delta j, \Delta(j+1)]}$ for a real sequence $\left.\left(c_{j}\right)_{j \in \mathbb{Z}}\right) \bar{F}_{\Delta} \in L^{2}([0, \Delta])$ is automatically satisfied and hence Theorem 1 recovers the results from Davis and Resnick (1985, Theorem 4.1), who derived the stable law as asymptotic distribution for the sample mean of discrete time moving averages $X_{t}=\sum_{j=0}^{\infty} c_{j} Z_{t-j}$ for $t \in \mathbb{Z}$. In contrast to their result, the parameters of the stable limit distribution are explicitly given in Theorem 2. However, we assume in addition to their assumptions that the distribution of $L_{1}$ is symmetric if $\alpha=1$.

(ii) Cohen and Lindner (2013) determined the asymptotic distribution of the sample mean for continuous time moving averages $X_{t}=\int_{\mathbb{R}} f(t-s) \mathrm{d} L_{s}$ under the assumption of $\mathbb{E}\left|L_{1}\right|^{2}<\infty$. They showed that

$$
\sqrt{n}\left(n^{-1} \sum_{t=1}^{n} X_{\Delta t}-\mathbb{E} X_{\Delta}\right) \stackrel{d}{\rightarrow} N\left(0, v^{2}\right)
$$


as $n \rightarrow \infty$ and for some explicitly given constant $v^{2}$, provided that $\bar{F}_{\Delta} \in L^{2}([0, \Delta])$. (iii) The condition $c_{\Delta}^{+}+c_{\Delta}^{-}=0$ of Theorem 1 (ii) applies if for example the kernel is given by $f(s)=\mathbb{1}_{[-1,0)}(s) \mathrm{e}^{-1-s}-\mathbb{1}_{[0,1)}(s) \mathrm{e}^{-s}$ when $\Delta=1$ and for arbitrary $p, q \in[0,1]$.

Define for $\Delta>0, h \in \mathbb{N}_{0}$ and $n \in \mathbb{N}$ the sample autocovariance function of $\left(X_{t}\right)_{t \in \mathbb{Z}}$ in (2.5) by $\gamma_{n, \Delta}(h)=n^{-1} \sum_{t=1}^{n} X_{\Delta t} X_{\Delta(t+h)}$ and

$$
G_{h, \Delta}:[0, \Delta] \rightarrow \mathbb{R}, u \mapsto \sum_{j=-\infty}^{\infty} f(\Delta j-u) f(\Delta(j+h)-u) .
$$

Theorem 3. Let $\Delta>0, h \in \mathbb{N}_{0}, \int_{\mathbb{R}}|f(s) f(s+\Delta h)| \mathrm{d} s>0$, conditions (A1) and (A2) be satisfied and set $X_{t}=\int_{-\infty}^{\infty} f(t-s) \mathrm{d} L_{s}$. Suppose $G_{0, \Delta} \in L^{\alpha / 2}([0, \Delta])$. Then $G_{h, \Delta} \in L^{\alpha / 2}([0, \Delta])$ for each $h \in \mathbb{N}_{0}$. Define the coefficients $c_{h, \Delta}^{+}$and $c_{h, \Delta}^{+}$by

$$
c_{h, \Delta}^{+}=\int_{0}^{\Delta}\left(G_{h, \Delta}^{+}(s)\right)^{\alpha / 2} \mathrm{~d} s, \quad c_{h, \Delta}^{-}=\int_{0}^{\Delta}\left(G_{h, \Delta}^{-}(s)\right)^{\alpha / 2} \mathrm{~d} s .
$$

(i) If $c_{h, \Delta}^{+}+c_{h, \Delta}^{-}>0$, then as $n \rightarrow \infty$

$$
\frac{n}{a_{n}^{2}} \gamma_{n, \Delta}(h)=\frac{1}{a_{n}^{2}} \sum_{t=1}^{n} X_{\Delta t} X_{\Delta(t+h)} \stackrel{d}{\rightarrow} S_{\alpha / 2}\left(\beta_{h, \Delta}, \gamma_{h, \Delta}, 0\right)
$$

where the parameters are given by

$$
\begin{aligned}
\beta_{h, \Delta} & =\frac{\int_{0}^{\Delta}\left(G_{h, \Delta}^{+}(s)\right)^{\alpha / 2}-\left(G_{h, \Delta}^{-}(s)\right)^{\alpha / 2} \mathrm{~d} s}{\int_{0}^{\Delta}\left|G_{h, \Delta}(s)\right|^{\alpha / 2} \mathrm{~d} s}, \\
\gamma_{h, \Delta}^{\alpha / 2} & =\left(c_{h, \Delta}^{+}+c_{h, \Delta}^{-}\right) \frac{\Gamma\left(2-\frac{\alpha}{2}\right)}{\left(1-\frac{\alpha}{2}\right)} \cos \left(\frac{\pi \alpha}{4}\right) .
\end{aligned}
$$

(ii) If $c_{h, \Delta}^{+}+c_{h, \Delta}^{-}=0$, then $\frac{n}{a_{n}^{2}} \gamma_{n, \Delta}(h) \stackrel{\mathbb{P}}{\rightarrow} 0$ as $n \rightarrow \infty$.

Remark 4. Theorem 3 recovers the result of Davis and Resnick (1985, Theorem 4.2) who applied point process techniques to derive the stable limit distribution for the autocovariance function of discrete moving averages. Again our method has the advantage that the parameters of the stable limit distribution are explicitly given.

Consider the special case of discrete time moving averages $\left(X_{n}\right)_{n \in \mathbb{N}}$, where for $Z_{j}=L_{j}-L_{j-1}, j \in \mathbb{Z}$, and $f(s)=\sum_{j=0}^{\infty} c_{j} \mathbb{1}_{[j, j+1)}(s),\left(c_{j}\right)_{j=0}^{\infty} \subset \mathbb{R}$,

$$
X_{n}=\int_{-\infty}^{\infty} f(n-s) \mathrm{d} L_{s}=\sum_{j=0}^{\infty} c_{j} Z_{n-j}, \quad n \in \mathbb{N} .
$$

Suppose that $\sum_{j=0}^{\infty}\left|c_{j}\right|^{\delta}<\infty$ for some $\delta<\alpha, \delta \leq 1$ and that the i.i.d. sequence $\left(Z_{j}\right)_{j \in \mathbb{Z}}$ satisfies for some slowly varying function $L:(0, \infty) \rightarrow(0, \infty)$ and $p, q \in$ $[0,1]$

$$
\begin{aligned}
& \mathbb{P}\left(\left|Z_{1}\right|>x\right)=L(x) x^{-\alpha}, \quad \alpha \in(0,2), \\
& \lim _{x \rightarrow \infty} \frac{\mathbb{P}\left(Z_{1}>x\right)}{\mathbb{P}\left(\left|Z_{1}\right|>x\right)}=p, \quad \lim _{x \rightarrow \infty} \frac{\mathbb{P}\left(Z_{1}<-x\right)}{\mathbb{P}\left(\left|Z_{1}\right|>x\right)}=q, \quad p+q=1 .
\end{aligned}
$$


Davis and Resnick (1985, Theorem 4.2) proved under these assumptions that for $\gamma_{n}(h):=n^{-1} \sum_{t=1}^{n} X_{t} X_{t+h}$ with $\left(X_{n}\right)_{n \in \mathbb{N}}$ in (3.4) and every $l \in \mathbb{N}$, as $n \rightarrow \infty$,

$$
\frac{n}{a_{n}^{2}}\left(\gamma_{n}(0), \ldots, \gamma_{n}(l)\right)^{T} \stackrel{d}{\rightarrow} Z_{\alpha / 2}\left(\sum_{j=0}^{\infty} c_{j}^{2}, \sum_{j=0}^{\infty} c_{j} c_{j+1}, \ldots, \sum_{j=0}^{\infty} c_{j} c_{j+l}\right)^{T},
$$

for a (positive) stable distributed random variable $Z_{\alpha / 2}$ with parameter $\alpha / 2$ and a positive sequence $\left(a_{n}\right)_{n \in \mathbb{N}}$ satisfying $n \mathbb{P}\left(\left|Z_{1}\right|>a_{n}\right) \rightarrow 1$ as $n \rightarrow \infty$. The following counter example shows that a similar statement does not hold for the more general case of continuous time moving averages.

Example 5. Let $\Delta=1, h \in \mathbb{N}_{0}$ and $\left(L_{t}\right)_{t \in \mathbb{R}}$ be a Lévy process satisfying condition (A1) with $p \neq \frac{1}{2}$. Consider the kernel $f(s)=2 \mathbb{1}_{\left(0, \frac{1}{2}\right]}(s)-\mathbb{1}_{\left(\frac{1}{2}, 1\right]}(s)+\mathbb{1}_{(1,2]}(s)$ which satisfies (A2). Then the assumptions of Theorem 3 are satisfied for $h=0,1$ and hence as $n \rightarrow \infty$

$$
\begin{aligned}
\frac{1}{a_{n}^{2}} \sum_{t=1}^{n} X_{t}^{2} & \stackrel{d}{\rightarrow} S_{\alpha / 2}\left(\beta_{0,1}, \gamma_{0,1}, 0\right), \\
\frac{1}{a_{n}^{2}} \sum_{t=1}^{n} X_{t} X_{t+1} & \stackrel{d}{\rightarrow} S_{\alpha / 2}\left(\beta_{1,1}, \gamma_{1,1}, 0\right),
\end{aligned}
$$

where the parameters $\gamma_{h, 1}$ are given in (3.3) and for $G_{h, 1}(s)=\sum_{j=-\infty}^{\infty} f(j+h-$ $s) f(j-s)$

$$
\beta_{h, 1}=\frac{\int_{0}^{1}\left(\left(G_{h, 1}^{+}(s)\right)^{\alpha / 2}-\left(G_{h, 1}^{-}(s)\right)^{\alpha / 2}\right) \mathrm{d} s}{\int_{0}^{1}\left|G_{h, 1}(s)\right|^{\alpha / 2} \mathrm{~d} s} .
$$

Notice that $G_{0,1} \geq 0$, so that $G_{0,1}=G_{0,1}^{+}=\left|G_{0,1}\right|$ and hence $\beta_{0,1}=1$. For $h=1$ and $s \in[0,1)$ we have $G_{1,1}(s)=f(1-s) f(2-s)$, and so $G_{1,1}^{+}(s)=$ $2 \mathbb{1}_{[1 / 2,1)}(s)$ and $G_{1,1}^{-}(s)=\mathbb{1}_{[0,1 / 2)}(s)$. Hence $\beta_{1,1}=\frac{2^{\alpha / 2}-1}{2^{\alpha / 2}+1}$. By eq. (3.2), $a X \sim$ $S_{\alpha / 2}(\operatorname{sign}(a) \beta,|a| \gamma, 0)$ for $X \sim S_{\alpha / 2}(\beta, \gamma, 0), \alpha<2$ and $a \in \mathbb{R} \backslash\{0\}$. But since both limit distributions (3.5) and (3.6) have different parameters $\beta_{0,1}, \beta_{1,1}$ with $\left|\beta_{0,1}\right| \neq\left|\beta_{1,1}\right|$ the joint convergence

$$
\frac{n}{a_{n}^{2}}\left(\gamma_{n, 1}(0), \gamma_{n, 1}(1)\right)^{T} \stackrel{d}{\rightarrow} Z_{\alpha / 2}\left(c_{f}(0), c_{f}(1)\right)^{T}
$$

cannot hold for a stable distributed random variable $Z_{\alpha / 2}$ with parameter $\alpha / 2$ and some constants $c_{f}(0)$ and $c_{f}(1)$ depending on the kernel $f$.

It is still possible that $\frac{n}{a_{n}^{2}}\left(\gamma_{n, 1}(0), \ldots, \gamma_{n, 1}(l)\right)^{T}$ converges for $l \in \mathbb{N}$ to a multivariate distribution, but we have not investigated the question of joint convergence further.

\section{Proofs}

In this section we prove our main results, Theorems 1 and 3 . We will apply in both proofs the following two Lévy-Itô decompositions (see Sato, 1999, Theorem 19.2). Suppose that condition (A1) is satisfied.

Decomposition (L1). The Lévy process $\left(L_{t}\right)_{t \in \mathbb{R}}$ admits the Lévy-Itô decomposition $L_{t}=L_{t}^{+}+L_{t}^{-}$for $t \in \mathbb{R}$, where $\left(L_{t}^{+}\right)_{t \in \mathbb{R}}$ and $\left(L_{t}^{-}\right)_{t \in \mathbb{R}}$ are independent Lévy processes such that $\left(L_{t}^{+}\right)_{t \in \mathbb{R}}$ has triplet $\left(\gamma, 0,\left.\nu\right|_{|x|>1}\right)$ and $\left(L_{t}^{-}\right)_{t \in \mathbb{R}}$ has triplet $\left(0, \sigma^{2},\left.\nu\right|_{|x| \leq 1}\right)$. 
The Lévy process $\left(L_{s}^{+}\right)_{s \in \mathbb{R}}$ is a compound Poisson processes (with drift) and admits therefore for $s \in[0,1]$ the representation $L_{s}^{+}=\gamma s+\sum_{i=1}^{N_{s}^{+}} \Delta L_{T_{i}^{+}}^{+}=\gamma s+\sum_{i=1}^{N_{s}^{+}} Y_{i}^{+}$, with jump times $\left(T_{i}^{+}\right)$and jump heights $\left(Y_{i}^{+}\right)$satisfying

$$
\mathbb{P}\left(Y_{i}^{+} \in \cdot\right)=\frac{\nu(\cdot \cap\{|x|>1\})}{\nu(\{|x|>1\})},
$$

and $\left(N_{s}^{+}\right)_{s \in[0,1]}$ being a Poisson process with parameters $\nu(\{|x|>1\})$, independent of $\left(T_{i}^{+}\right)$and $\left(Y_{i}^{+}\right)$.

Decomposition (L2). The Lévy process $\left(L_{t}^{+}\right)_{t \in \mathbb{R}}$ in (L1) admits for a sequence $\left(a_{n}\right)_{n \in \mathbb{N}}$ satisfying (2.1) the further decomposition into $L_{t}^{+}=L_{t}^{\leq, n}+L_{t}^{>, n}$ for $t \in \mathbb{R}$, where $\left(L_{t}^{\leq, n}\right)_{t \in \mathbb{R}}$ and $\left(L_{t}^{>}, n\right)_{t \in \mathbb{R}}$ are independent Lévy processes with triplets $\left(\gamma, 0,\left.\nu\right|_{1<|x| \leq a_{n}}\right)$ and $\left(0,0,\left.\nu\right|_{|x|>a_{n}}\right)$, respectively. We usually suppress the index $\mathrm{n}$ and simply write $L_{t}^{\leq}$for $L_{t}^{\leq, n}$ and $L_{t}^{>}$for $L_{t}^{>, n}$. The Lévy processes $\left(L_{s}^{\leq}\right)_{s \in \mathbb{R}}$ and $\left(L_{s}^{>}\right)_{s \in \mathbb{R}}$ are compound Poisson processes (with drift) and admit therefore for $s \in[0,1]$ the representations

$$
L_{s}^{\leq}=\gamma s+\sum_{i=1}^{N_{s}^{\leq}} \Delta L_{T_{i}^{\leq}}^{\leq}=\gamma s+\sum_{i=1}^{N_{s}^{\leq}} Y_{i, n}^{\leq}, \quad L_{s}^{>}=\sum_{i=1}^{N_{s}^{>}} \Delta L_{T_{i}^{>}}^{>}=\sum_{i=1}^{N_{s}^{>}} Y_{i, n}^{>},
$$

with jump times $\left(T_{i}^{\leq}\right)$and $\left(T_{i}^{>}\right)$and jump heights $\left(Y_{i, n}^{\leq}\right)$and $\left(Y_{i, n}^{>}\right)$satisfying

$$
\mathbb{P}\left(Y_{i, n}^{\leq} \in \cdot\right)=\frac{\nu\left(\cdot \cap\left\{1<|x| \leq a_{n}\right\}\right)}{\nu\left(\left\{1<|x| \leq a_{n}\right\}\right)}, \quad \mathbb{P}\left(Y_{i, n}^{>} \in \cdot\right)=\frac{\nu\left(\cdot \cap\left\{|x|>a_{n}\right\}\right)}{\nu\left(\left\{|x|>a_{n}\right\}\right)},
$$

and $\left(N_{s}^{\leq}\right)_{s \in[0,1]},\left(N_{s}^{>}\right)_{s \in[0,1]}$ being Poisson processes with parameters $\nu(\{1<|x| \leq$ $\left.\left.a_{n}\right\}\right)$ and $\nu\left(\left\{|x|>a_{n}\right\}\right)$, independent of $\left(T_{i}^{\leq}\right)$and $\left(Y_{i, n}^{\leq}\right)$and of $\left(T_{i}^{>}\right)$and $\left(Y_{i, n}^{>}\right)$, respectively.

By a time change we can restrict ourselves in the following proofs to the case $\Delta=1$ since we can write for $g_{\Delta}(x):=f(\Delta x)$ and the Lévy process $\left(U_{v}^{\Delta}\right)_{v \in \mathbb{R}}$ with $U_{v}^{\Delta}:=L_{\Delta v}$

$$
X_{\Delta t}=\int_{\mathbb{R}} f(\Delta t-s) \mathrm{d} L_{s}=\int_{\mathbb{R}} g_{\Delta}(t-v) \mathrm{d} U_{v}^{\Delta}=: X_{t}^{\Delta}, \quad t \in \mathbb{R},
$$

where $\left(X_{t}^{\Delta}\right)_{t \in \mathbb{R}}$ is observed on the lattice $(t)_{t \in \mathbb{Z}}$ whereas $\left(X_{t}\right)_{t \in \mathbb{R}}$ is observed on $(\Delta t)_{t \in \mathbb{Z}}$. In the proof of the following proposition we will use the notion of vague convergence and in particular that the vague convergence $\mu_{n} \stackrel{v}{\rightarrow} \mu$ of a sequence of Radon measures $\left(\mu_{n}\right)_{n \in \mathbb{N}}, \mu$ on $\left(\overline{\mathbb{R}}^{d} \backslash\{0\}, \mathcal{B}\left(\overline{\mathbb{R}}^{d} \backslash\{0\}\right)\right)$ is equivalent to $\mu_{n}(B) \rightarrow \mu(B)$ as $n \rightarrow \infty$, for all relatively compact sets $B \in \mathcal{B}\left(\overline{\mathbb{R}}^{d} \backslash\{0\}\right)$ such that $\mu(\partial B)=0$ (see Kallenberg, 1983, Theorem 15.7.2). Notice that the relatively compact sets in $\mathcal{B}\left(\overline{\mathbb{R}}^{d} \backslash\{0\}\right)$ are the sets $B \in \mathcal{B}\left(\overline{\mathbb{R}}^{d} \backslash\{0\}\right)$ which are bounded away from zero, i.e. $0 \notin \overline{B \cap \mathbb{R}^{d}}$.

Proposition 1. Let $\left(L_{t}\right)_{t \in \mathbb{R}}$ be a Lévy process and $f: \mathbb{R} \rightarrow \mathbb{R}$ a function such that (A1) and (A2) are satisfied. Then for $\left(X_{t}\right)_{t \in \mathbb{R}}$ in (2.5) the following statements hold:

(i)[Moser and Stelzer, 2011, Theorem 3.1] The random vector $\boldsymbol{X}_{k}=\left(X_{1}, \ldots, X_{k}\right)^{T}$ 
satisfies $\boldsymbol{X}_{k} \in R V\left(\alpha, a_{n}, \mu_{f_{k}}\right)$ for each $k \in \mathbb{N}$, where for $f_{k}(s):=(f(1-s), f(2-$ $s), \ldots, f(k-s))^{T}$ the measure $\mu_{f_{k}}$ is given by

$$
\mu_{f_{k}}(B)=\int_{-\infty}^{\infty} \int_{-\infty}^{\infty} \mathbb{1}_{B}\left(f_{k}(s) x\right) \mu(\mathrm{d} x) \mathrm{d} s, \quad B \in \mathcal{B}\left(\overline{\mathbb{R}}^{k} \backslash\{0\}\right) .
$$

(ii) For $k \in \mathbb{N}$ and $h \in \mathbb{N}_{0}$ define $g_{k, h}: \mathbb{R}^{k+h} \rightarrow \mathbb{R}^{k}, g_{k, h}\left(x_{1}, \ldots, x_{k+h}\right)=$ $\left(x_{1} x_{1+h}, \ldots, x_{k} x_{k+h}\right)^{T}$. Then $\boldsymbol{Z}_{k, h}:=g_{k, h}\left(\boldsymbol{X}_{k+h}\right)=\left(X_{1} X_{1+h}, \ldots, X_{k} X_{k+h}\right)^{T} \in$ $R V\left(\alpha / 2, a_{n}^{2}, \mu_{f_{k+h}} \circ g_{k, h}^{-1}\right)$.

Denote by $\AA$ and $\bar{A}$ the interior and closure of a set $A$, respectively.

Proof: (ii) The function $g_{k, h}$ is continuous, 2-homogeneous (i.e. $g_{k, h}(t x)=t^{2} g_{k, h}(x)$ for $t>0$ and $x \in \mathbb{R}^{k+h}$ ) and $g_{k, h}^{-1}(A)$ is bounded away from zero (i.e. $0 \notin \overline{g_{k, h}^{-1}(A)}$ ) for every set $A \in \mathcal{B}\left(\overline{\mathbb{R}}^{k} \backslash\{0\}\right)$, which is bounded away from zero. To prove this claim, assume $A$ is bounded away from zero, but $0 \in \overline{g_{k, h}^{-1}(A)}$. Then there is a sequence $\left(x_{n}\right)_{n \in \mathbb{N}} \subset g_{k, h}^{-1}(A)$ such that $x_{n} \rightarrow 0$ as $n \rightarrow \infty$. But then for $\left(g_{k, h}\left(x_{n}\right)\right)_{n \in \mathbb{N}} \subset A$ it holds that $g_{k, h}\left(x_{n}\right) \rightarrow 0$ (since $g_{k, h}$ is continuous) implying $0 \in \bar{A}$ which is a contradiction. Therefore, as $x \rightarrow \infty$, and for $A \in \mathcal{B}\left(\overline{\mathbb{R}}^{k} \backslash\{0\}\right)$ bounded away from zero such that $\mu_{f_{k+h}} \circ g_{k, h}^{-1}(\partial A)=0$

$$
\begin{aligned}
n \mathbb{P}\left(a_{n}^{-2} \boldsymbol{Z}_{k, h} \in A\right) & =n \mathbb{P}\left(a_{n}^{-2} g_{k, h}\left(\boldsymbol{X}_{k+h}\right) \in A\right)=n \mathbb{P}\left(g_{k, h}\left(a_{n}^{-1} \boldsymbol{X}_{k+h}\right) \in A\right) \\
& =n \mathbb{P}\left(a_{n}^{-1} \boldsymbol{X}_{k+h} \in g_{k, h}^{-1}(A)\right) \rightarrow \mu_{f_{k+h}}\left(g_{k, h}^{-1}(A)\right),
\end{aligned}
$$

i.e. $n \mathbb{P}\left(a_{n}^{-2} \boldsymbol{Z}_{k, h} \in A\right) \stackrel{v}{\rightarrow} \mu_{f_{k+h}} \circ g_{k, h}^{-1}$. Notice that we used here that $\overline{g_{k, h}^{-1}(A)} \subset$ $g_{k, h}^{-1}(\bar{A})$ and $g_{k, h}^{-1}(\AA) \subset\left[g_{k, h}^{-1}(A)\right]^{\circ}$, and hence $\mu_{f_{k+h}} \circ g_{k, h}^{-1}(\partial A)=0$ implies that also $\mu_{f_{k+h}}\left(\partial g_{k, h}^{-1}(A)\right)=0$ since

$$
\begin{aligned}
0=\mu_{f_{k+h}}\left(g_{k, h}^{-1}(\partial A)\right)=\mu_{f_{k+h}}\left(g_{k, h}^{-1}(\bar{A} \backslash \AA)\right) & =\mu_{f_{k+h}}\left(g_{k, h}^{-1}(\bar{A})\right)-\mu_{f_{k+h}}\left(g_{k, h}^{-1}(\AA)\right) \\
& \geq \mu_{f_{k+h}}\left(\overline{g_{k, h}^{-1}(A)}\right)-\mu_{f_{k+h}}\left(\left[g_{k, h}^{-1}(A)\right]^{\circ}\right) \\
& =\mu_{f_{k+h}}\left(\partial g_{k, h}^{-1}(A)\right) \geq 0 .
\end{aligned}
$$

Next we will consider strictly stationary sequences $\left(X_{t}\right)_{t \in \mathbb{Z}}$ which are regularly varying in the following sense: for every $d \in \mathbb{N}$ and some (equivalently: every) sequence $\left(r_{n}\right)_{n \in \mathbb{N}}$ satisfying $r_{n} \rightarrow \infty$ and

$$
n \mathbb{P}\left(\left|X_{1}\right| \geq r_{n}\right) \rightarrow 1, \quad(n \rightarrow \infty)
$$

there exists a non-null Radon measure $\mu_{d}$ on $\left(\overline{\mathbb{R}}^{d} \backslash\{0\}, \mathcal{B}\left(\overline{\mathbb{R}}^{d} \backslash\{0\}\right)\right)$ with $\mu_{d}\left(\overline{\mathbb{R}}^{d} \backslash \mathbb{R}^{d}\right)$ $=0$ such that

$$
n \mathbb{P}\left(r_{n}^{-1}\left(X_{1}, \ldots, X_{d}\right)^{T} \in \cdot\right) \stackrel{v}{\rightarrow} \mu_{d}(\cdot) \quad(n \rightarrow \infty) .
$$

The measure $\mu_{d}$ does not depend on the specific choice of the sequence $\left(r_{n}\right)_{n \in \mathbb{N}}$. Notice that under (A1) and (A2) for $I:=\left(\int_{\mathbb{R}}|f(s)|^{\alpha} \mathrm{d} s\right)^{1 / \alpha}$ and $r_{n}:=I a_{n}$ by Proposition 1(i)

$$
\lim _{n \rightarrow \infty} n \mathbb{P}\left(\left|X_{1}\right| \geq r_{n}\right)=\lim _{n \rightarrow \infty} n \mathbb{P}\left(\left|a_{n}^{-1} X_{1}\right|>I\right)=\mu_{f_{1}}((I, \infty) \cup(-\infty,-I))=1 .
$$


Hence under (A1) and (A2) the sequence $\left(X_{t}\right)_{t \in \mathbb{Z}}$ in (2.5) is regularly varying in the sense of (4.4) with $r_{n}=\left(\int_{\mathbb{R}}|f(s)|^{\alpha} \mathrm{d} s\right)^{1 / \alpha} a_{n}$ satisfying (4.3) and $\mu_{d}:=$ $\mu_{f_{d}} /\left(\int|f(s)|^{\alpha} \mathrm{d} s\right)$ with $\mu_{f_{d}}$ in (4.2). Observe that if we scale instead by $\left(a_{n}\right)_{n \in \mathbb{N}}$ satisfying (2.1), then $n \mathbb{P}\left(a_{n}^{-1}\left(X_{1}, \ldots, X_{d}\right)^{T} \in \cdot\right) \stackrel{v}{\rightarrow} \mu_{f_{d}}(\cdot)$. Define further for $\left(r_{n}\right)_{n \in \mathbb{N}}$ satisfying (4.3), a sequence $\left(X_{t}\right)_{t \in \mathbb{Z}}$ satisfying (4.4) and $S_{n}=\sum_{i=1}^{n} X_{i}$ the parameters

$$
y^{+}(d)=\lim _{n \rightarrow \infty} n \mathbb{P}\left(S_{d}>r_{n}\right), \quad y^{-}(d)=\lim _{n \rightarrow \infty} n \mathbb{P}\left(S_{d}<-r_{n}\right), \quad d \geq 1 .
$$

We will show later for $\left(X_{t}\right)_{t \in \mathbb{Z}}$ defined in (2.5) the existence of these limits (see the proof of Proposition 3). For general sequences $\left(X_{t}\right)_{t \in \mathbb{Z}}$ satisfying (4.3) and (4.4), a similar argument can be given to show existence of $y^{+}(d)$ and $y^{-}(d)$. Our proofs of the main results will rely on the following limit theorem for strictly stationary, $m$-dependent and regularly varying sequences.

Proposition 2 (Bartkiewicz et al., 2011, Proposition 1). Assume that $\left(X_{t}\right)_{t \in \mathbb{Z}}$ is a strictly stationary, $m$-dependent sequence for some $m \geq 1$ which satisfies (4.4) with a sequence $\left(r_{n}\right)_{n \in \mathbb{N}}$ satisfying (4.3) for some $\alpha \in(0,2)$. Moreover, assume $\mathbb{E} X_{1}=0$ if $\alpha>1$ and for $\alpha=1$ that the distribution of $X_{1}$ is symmetric. Then $z_{m}^{+}, z_{m}^{-} \geq 0$, where for $y^{+}(d), y^{-}(d)$ in $(4.5)$

$$
z_{m}^{+}=y^{+}(m+1)-y^{+}(m), \quad z_{m}^{-}=y^{-}(m+1)-y^{-}(m) .
$$

(i) If $z_{m}^{+}+z_{m}^{-}>0$, then $r_{n}^{-1} S_{n} \stackrel{d}{\rightarrow} S_{\alpha}\left(\tau_{m}, \lambda_{m}, 0\right)$ as $n \rightarrow \infty$, where

$$
\tau_{m}=\frac{z_{m}^{+}-z_{m}^{-}}{z_{m}^{+}+z_{m}^{-}}, \quad \lambda_{m}^{\alpha}= \begin{cases}\left(z_{m}^{+}+z_{m}^{-}\right) \frac{\Gamma(2-\alpha)}{(1-\alpha)} \cos \left(\frac{\pi \alpha}{2}\right), & \alpha \neq 1, \\ \left(z_{m}^{+}+z_{m}^{-}\right) \frac{\pi}{2}, & \alpha=1 .\end{cases}
$$

(ii) If $z_{m}^{+}+z_{m}^{-}=0$, then $r_{n}^{-1} S_{n} \stackrel{\mathbb{P}}{\rightarrow} 0$ as $n \rightarrow \infty$.

Let $\left(L_{t}\right)_{t \in \mathbb{R}}$ and $f$ satisfy the conditions (A1) and (A2) and define for $m \in \mathbb{N}$ and $\Delta>0$ the compactly supported function $f_{\Delta m}(s)=f(s) \mathbb{1}_{[-\Delta m, \Delta m]}(s)$ and the sequence $\left(X_{t, \Delta m}\right)_{t \in \mathbb{Z}}$, defined by

$$
X_{t, \Delta m}:=\int_{-\infty}^{\infty} f_{\Delta m}(t-s) \mathrm{d} L_{s}=\int_{t-\Delta m}^{t+\Delta m} f(t-s) \mathrm{d} L_{s}, \quad t \in \mathbb{R} .
$$

We will use the more natural scaling by $\left(a_{n}\right)_{n \in \mathbb{N}}$ satisfying (2.1) instead of the scaling $\left(r_{n}\right)_{n \in \mathbb{N}}$ in (4.3). The advantage of this scaling is that it depends only on the Lévy process $\left(L_{t}\right)_{t \in \mathbb{R}}$ and not on the kernel $f$ nor on $\Delta>0$ and $m \in \mathbb{N}$. Applying Proposition 2 to the sequence $\left(X_{\Delta t, \Delta m}\right)_{t \in \mathbb{Z}}$ yields the following limit theorem for its sample mean.

Proposition 3. Let (A1) and (A2) be satisfied and assume that the distribution of $L_{1}$ is symmetric if $\alpha=1$. Let $\Delta>0$ and $m \in \mathbb{N}$ sufficiently large, so that $\int_{\mathbb{R}}\left|f_{\Delta m}(s)\right| \mathrm{d} s>0$. Then for $\left(X_{t, \Delta m}\right)_{t \in \mathbb{Z}}$ in (4.7), $S_{n, \Delta, m}=\sum_{t=1}^{n} X_{\Delta t, \Delta m}$ and $d \geq 1$ the limits

$$
b_{\Delta, m}^{+}(d)=\lim _{n \rightarrow \infty} n \mathbb{P}\left(S_{d, \Delta, m}>a_{n}\right), \quad b_{\Delta, m}^{-}(d)=\lim _{n \rightarrow \infty} n \mathbb{P}\left(S_{d, \Delta, m}<-a_{n}\right),
$$


exist and the parameters $c_{\Delta, m}^{ \pm}=b_{\Delta, m}^{ \pm}(2 m)-b_{\Delta, m}^{ \pm}(2 m-1)$ are given by

$$
\begin{aligned}
& c_{\Delta, m}^{+}=\int_{0}^{\Delta}\left(p\left[\left(\sum_{t=1-m}^{m} f(\Delta t-s)\right)^{+}\right]^{\alpha}+q\left[\left(\sum_{t=1-m}^{m} f(\Delta t-s)\right)^{-}\right]^{\alpha}\right) \mathrm{d} s, \\
& c_{\Delta, m}^{-}=\int_{0}^{\Delta}\left(q\left[\left(\sum_{t=1-m}^{m} f(\Delta t-s)\right)^{+}\right]^{\alpha}+p\left[\left(\sum_{t=1-m}^{m} f(\Delta t-s)\right)^{-}\right]^{\alpha}\right) \mathrm{d} s .
\end{aligned}
$$

(i) If $c_{\Delta, m}^{+}+c_{\Delta, m}^{-}>0$, then $a_{n}^{-1} S_{n, \Delta, m} \stackrel{d}{\rightarrow} S_{\alpha}\left(\beta_{\Delta, m} \gamma_{\Delta, m}, 0\right)$ for $n \rightarrow \infty$, where the parameters are given by

$$
\beta_{\Delta, m}=\frac{c_{\Delta, m}^{+}-c_{\Delta, m}^{-}}{c_{\Delta, m}^{+}+c_{\Delta, m}^{-}}, \quad \gamma_{\Delta, m}^{\alpha}= \begin{cases}\left(c_{\Delta, m}^{+}+c_{\Delta, m}^{-}\right) \frac{\Gamma(2-\alpha)}{(1-\alpha)} \cos \left(\frac{\pi \alpha}{2}\right), & \alpha \neq 1, \\ \left(c_{\Delta, m}^{+}+c_{\Delta, m}^{-}\right) \frac{\pi}{2}, & \alpha=1 .\end{cases}
$$

(ii) If $c_{\Delta, m}^{+}+c_{\Delta, m}^{-}=0$, then $a_{n}^{-1} S_{n, \Delta, m} \stackrel{\mathbb{P}}{\rightarrow} 0$ as $n \rightarrow \infty$.

Proof: W.l.o.g we suppose $\Delta=1$ (the other cases follow from a time change, see (4.1)). By Proposition 1 (i), $X_{1, m} \in R V\left(\alpha, a_{n}, \mu_{f_{1}^{(m)}}\right)$ where for $f_{d}^{(m)}:=\left(f_{m}(1-\right.$ $\left.s), \ldots, f_{m}(d-s)\right)^{T}$ the measure $\mu_{f_{1}^{(m)}}$ is defined in (4.2). Denote $\varphi\left(x_{1}, \ldots, x_{d}\right)=$ $\sum_{i=1}^{d} x_{i}$ and notice that $\varphi^{-1}((1, \infty))$ is bounded away from zero. Hence

$$
\begin{aligned}
b_{1, m}^{+}(d) & =\lim _{n \rightarrow \infty} n \mathbb{P}\left(S_{d, 1, m}>a_{n}\right) \\
& =\lim _{n \rightarrow \infty} n \mathbb{P}\left(a_{n}^{-1}\left(X_{1, m}, \ldots, X_{d, m}\right)^{T} \in \varphi^{-1}((1, \infty))\right) \\
& =\mu_{f_{d}^{(m)}}\left(\varphi^{-1}((1, \infty))\right),
\end{aligned}
$$

provided $\mu_{f_{d}^{(m)}}\left(\partial \varphi^{-1}((1, \infty))\right)=0$. This is the case since by continuity of $\varphi$ and $(4.2)$

$$
\begin{aligned}
\mu_{f_{d}^{(m)}}\left(\partial \varphi^{-1}((1, \infty))\right) & \leq \mu_{f_{d}^{(m)}}\left(\varphi^{-1}(\partial(1, \infty))\right)=\mu_{f_{d}^{(m)}}\left(\varphi^{-1}(\{1\})\right) \\
& =\int_{\mathbb{R}} \mu\left(\left\{\left(\sum_{i=1}^{d} f_{m}(i-s)\right)^{-1}\right\}\right) \mathrm{d} s=0 .
\end{aligned}
$$

Hence the coefficients $b_{1, m}^{+}$and $b_{1, m}^{-}$are well-defined. By Proposition 1 (i) and the discussion after eq. (4.4), $\left(X_{t, m}\right)_{t \in \mathbb{Z}}$ in (4.7) is regularly varying in the sense of (4.4) with $\mu_{d}=\mu_{f_{d}^{(m)}} /\left(\int\left|f_{m}(s)\right|^{\alpha} \mathrm{d} s\right)$ and $r_{n}=\left(\int_{\mathbb{R}}\left|f_{m}(s)\right|^{\alpha} \mathrm{d} s\right)^{1 / \alpha} a_{n}$ satisfying (4.3). Observe that the sequence $\left(X_{t, m}\right)_{t \in \mathbb{Z}}$ is $(2 m-1)$-dependent, i.e. $\left(X_{s, m}\right)_{s \leq t}$ is independent of $\left(X_{s, m}\right)_{s \geq t+2 m}$ for every $t \in \mathbb{Z}$. Hence we checked that $\left(X_{t, m}\right)_{t \in \mathbb{Z}}$ satisfies the assumptions of Proposition 2 with $r_{n}=\left(\int\left|f_{m}(s)\right|^{\alpha} \mathrm{d} s\right)^{1 / \alpha} a_{n}$. Let $y^{ \pm}$ denote the coefficients in (4.5) corresponding to the partial sums of $\left(X_{t, m}\right)_{t \in \mathbb{Z}}$, i.e.

$$
y^{+}(d)=\lim _{n \rightarrow \infty} n \mathbb{P}\left(S_{d, 1, m}>r_{n}\right), \quad y^{-}(d)=\lim _{n \rightarrow \infty} n \mathbb{P}\left(S_{d, 1, m}<-r_{n}\right), \quad d \geq 1 .
$$

Then $y^{ \pm}(d)=b_{1, m}^{ \pm}(d) /\left(\int_{\mathbb{R}}\left|f_{m}(s)\right|^{\alpha} \mathrm{d} s\right)$ and if $c_{1, m}^{+}+c_{1, m}^{-}>0$ by Proposition 2

$$
r_{n}^{-1} S_{n, 1, m} \stackrel{d}{\rightarrow} S_{\alpha}\left(\tau_{m}, \lambda_{m}, 0\right), \quad n \rightarrow \infty,
$$

where the parameters are given by (4.6). But since $z_{m}^{ \pm}(d)\left(\int_{\mathbb{R}}\left|f_{m}(s)\right|^{\alpha} \mathrm{d} s\right)=b_{1, m}^{ \pm}(d)$ and by (3.2), it follows that $a_{n}^{-1} S_{n, 1, m} \stackrel{d}{\rightarrow} S_{\alpha}\left(\beta_{1, m} \gamma_{1, m}, 0\right)$ with the parameters 
given in (4.11). The statement for the case $c_{1, m}^{+}+c_{1, m}^{-}=0$ is clear. It remains to prove (4.9) and (4.10). Notice that for $m \in \mathbb{N}^{2}$

$$
\sum_{t=1}^{2 m} X_{t, m}=\int_{-\infty}^{\infty}\left(\sum_{t=1}^{2 m} f_{m}(t-s)\right) \mathrm{d} L_{s}=\sum_{i=1-m}^{3 m-1} \sum_{t=1 \vee(i-m+1)}^{2 m \wedge(i+m)} \int_{i}^{i+1} f(t-s) \mathrm{d} L_{s} .
$$

Recall the definition of $\mu$ in $(2.2)$ and notice that $x f_{2 m}^{(m)}(s) \in \varphi^{-1}((1, \infty))$ if and only if $x \sum_{i=1}^{2 m} f_{m}(i-s) \geq 1$. Hence we conclude by (4.12) that

$$
\begin{aligned}
b_{1, m}^{+}(2 m) & =\mu_{f_{2 m}^{(m)}}\left(\varphi^{-1}((1, \infty))\right) \\
& =\int_{-\infty}^{\infty} p\left(\left(\sum_{t=1}^{2 m} f_{m}(t-s)\right)^{+}\right)^{\alpha}+q\left(\left(\sum_{t=1}^{2 m} f_{m}(t-s)\right)^{-}\right)^{\alpha} \mathrm{d} s \\
& =\sum_{i=1-m}^{3 m-1} \int_{i}^{i+1} p\left(\left(\sum_{t=1 \vee(i-m+1)}^{2 m \wedge(i+m)} f(t-s)\right)^{+}\right)^{\alpha}+q\left(\left(\sum_{t=1 \vee(i-m+1)}^{2 m \wedge(i+m)} f(t-s)\right)^{-}\right)^{\alpha} \mathrm{d} s .
\end{aligned}
$$

Similarly, one calculates $b_{1, m}^{+}(2 m-1), b_{1, m}^{-}(2 m)$ and $b_{1, m}^{-}(2 m-1)$. For simplification of notation assume that $f=f^{+}$. Notice that in this case

$$
\begin{aligned}
p^{-1} b_{1, m}^{+}(2 m) & =\sum_{i=1-m}^{3 m-1} \int_{i}^{i+1}\left(\sum_{t=1 \vee(i-m+1)}^{2 m \wedge(i+m)} f(t-s)\right)^{\alpha} \mathrm{d} s \\
& =\sum_{i=1}^{2 m-1} \int_{0}^{1}\left(\sum_{t=1+m}^{i+m} f(t-s-i)\right)^{\alpha} \mathrm{d} s+\sum_{i=0}^{2 m-1} \int_{0}^{1}\left(\sum_{t=1-m}^{m-i} f(t-s)\right)^{\alpha} \mathrm{d} s .
\end{aligned}
$$

Similarly,

$$
p^{-1} b_{1, m}^{+}(2 m-1)=\sum_{i=1}^{2 m-1} \int_{0}^{1}\left(\sum_{t=1+m}^{i+m} f(t-s-i)\right)^{\alpha} \mathrm{d} s+\sum_{i=1}^{2 m-1} \int_{0}^{1}\left(\sum_{t=1-m}^{m-i} f(t-s)\right)^{\alpha} \mathrm{d} s .
$$

Hence we conclude

$$
p^{-1}\left(b_{1, m}^{+}(2 m)-b_{1, m}^{+}(2 m-1)\right)=\int_{0}^{1}\left(\sum_{t=1-m}^{m} f(t-s)\right)^{\alpha} \mathrm{d} s .
$$

Similar calculations for $f^{-}$yield (4.9) and (4.10).

Proof of Theorem 1: For simplicity in notation we assume $\Delta=1$ (the other cases follow from a time change, see (4.1)). By the Lévy-Itô decomposition (L1), $L_{t}=$ $L_{t}^{+}+L_{t}^{-}$for $t \in \mathbb{R}$. Denote $X_{t, m}^{+}=\int_{-\infty}^{\infty} f_{m}(t-s) \mathrm{d} L_{s}^{+}$and $X_{t}^{ \pm}=\int_{-\infty}^{\infty} f(t-s) \mathrm{d} L_{s}^{ \pm}$. Then $a_{n}^{-1} \sum_{t=1}^{n} X_{t}^{-}$converges in probability to zero as $n \rightarrow \infty$ by Remark 2 (ii) since $\mathbb{E} X_{t}^{-}=\mathbb{E} L_{1}^{-}=0$ and $\sqrt{n} / a_{n} \rightarrow 0$ by (2.4). Hence $d-\lim _{n \rightarrow \infty} a_{n}^{-1} \sum_{t=1}^{n} X_{t}=$ $d-\lim _{n \rightarrow \infty} a_{n}^{-1} \sum_{t=1}^{n} X_{t}^{+}$. By Hölder's inequality

$$
\int_{0}^{1}\left|\bar{F}_{1}(s)\right|^{\alpha} \mathrm{d} s \leq\left(\int_{0}^{1}\left|\bar{F}_{1}(s)\right|^{2} \mathrm{~d} s\right)^{\alpha / 2}<\infty
$$

and thus by the Theorem of Lebesgue the limits $c_{1}^{ \pm}:=\lim _{m \rightarrow \infty} c_{1, m}^{ \pm}$of the quantities $c_{1, m}^{+}$and $c_{1, m}^{-}$, defined in (4.9)-(4.10), exist and are given by

$$
c_{1}^{+}=\int_{0}^{1}\left(p\left(F_{1}^{+}(s)\right)^{\alpha}+q\left(F_{1}^{-}(s)\right)^{\alpha}\right) \mathrm{d} s, \quad c_{1}^{-}=\int_{0}^{1}\left(q\left(F_{1}^{+}(s)\right)^{\alpha}+p\left(F_{1}^{-}(s)\right)^{\alpha}\right) \mathrm{d} s .
$$


Observe that $c_{1, m}^{+}+c_{1, m}^{-}>0$ for large $m \in \mathbb{N}$ provided $c_{1}^{+}+c_{1}^{-}>0$ and also $\int\left|f_{m}(s)\right|^{\alpha} \mathrm{d} s>0$ for sufficiently large $m$. Hence if $c_{1}^{+}+c_{1}^{-}>0$, for large enough $m$ by Proposition 3

$$
\frac{1}{a_{n}} \sum_{t=1}^{n} X_{t, m}^{+} \stackrel{d}{\rightarrow} S_{\alpha}\left(\beta_{1, m}, \gamma_{1, m}, 0\right) \quad(n \rightarrow \infty),
$$

where the parameters are given in (4.11). Applying the second Lévy-Itô decomposition (L2) such that $L_{t}^{+}=L_{t}^{\leq}+L_{t}^{>}$we define for $t \in \mathbb{N}$ and $k \in \mathbb{Z}$

$$
Z_{t, k}^{\leq}:=\int_{k}^{k+1} f(t-s) \mathrm{d} L_{s}^{\leq}, \quad Z_{t, k}^{>}:=\int_{k}^{k+1} f(t-s) \mathrm{d} L_{s}^{>}, \quad \widetilde{Z}_{t, k}^{\leq}:=Z_{t, k}^{\leq}-\mathbb{E} Z_{t, k}^{\leq} .
$$

Next we want to prove that, as $n \rightarrow \infty$,

$$
\frac{1}{a_{n}}\left(\sum_{t=1}^{n} X_{t, m}^{+}-n \sum_{j=-m}^{m-1} \mathbb{E} Z_{0, j}^{\leq}\right) \stackrel{d}{\rightarrow} S_{\alpha}\left(\beta_{1, m}, \gamma_{1, m}, \delta_{1, m}\right),
$$

where $\delta_{1, m}=-\frac{\alpha(p-q)}{1-\alpha} \int_{-m}^{m} f(-s) \mathrm{d} s$. To this end let first $\alpha \in(0,1)$. Using the compound Poisson representation and since $\lim _{n \rightarrow \infty} n a_{n}^{-1} \gamma \int_{0}^{1} f(-j-s) \mathrm{d} s=0$ we have

$$
\lim _{n \rightarrow \infty} \frac{n}{a_{n}} \mathbb{E} Z_{0, j}^{\leq}=\lim _{n \rightarrow \infty} \frac{n}{a_{n}} \mathbb{E} \int_{0}^{1} f(-j-s) \mathrm{d} L_{s}^{\leq}=\lim _{n \rightarrow \infty} \frac{n}{a_{n}} \mathbb{E}\left(\sum_{k=1}^{N_{1}^{\leq}} f\left(-j-T_{k}^{\leq}\right) Y_{k, n}^{\leq}\right) .
$$

Conditioning on the number of jumps and jump times yields for $p_{n}:=\mathbb{P}\left(N_{1}^{\leq}=n\right)$

$$
\begin{aligned}
& \mathbb{E}\left(\sum_{k=1}^{N_{1}^{\leq}} f\left(-j-T_{k}^{\leq}\right) Y_{k, n}^{\leq}\right) \\
& =\mathbb{E} Y_{1, n}^{\leq} \sum_{N=0}^{\infty} p_{N} \int_{\mathbb{R}^{N}}\left(\sum_{k=1}^{N} f\left(-j-\sum_{l=1}^{k} t_{l}\right)\right) \mathbb{P}\left(T_{1}^{\leq}-T_{0}^{\leq}=\mathrm{d} t_{1}, \ldots, T_{N}^{\leq}-T_{N}^{\leq}=\mathrm{d} t_{N}\right) \\
& =\mathbb{E} Y_{1, n}^{\leq} \mathbb{E} \int_{0}^{1} f(-j-s) \mathrm{d} N_{s}^{\leq}=\mathbb{E} Y_{1, n}^{\leq} \mathbb{E} N_{1}^{\leq} \int_{0}^{1} f(-j-s) \mathrm{d} s .
\end{aligned}
$$

By Bingham et al. (1989, Theorem 1.6.4) and since $\mathbb{E} N_{1}^{\leq}=\nu\left(1<|x| \leq a_{n}\right)$

$$
\lim _{n \rightarrow \infty} \frac{n \mathbb{E} N_{1}^{\leq} \mathbb{E} Y_{1, n}^{\leq}}{a_{n}}=-\lim _{n \rightarrow \infty} \frac{n}{a_{n}} \int_{1<x \leq a_{n}} x \bar{\nu}(\mathrm{d} x)=\frac{\alpha(p-q)}{(1-\alpha)} .
$$

If $\alpha \in(1,2)$ we assumed $\mathbb{E} L_{1}=0$, hence $\mathbb{E} L_{1}^{+}=0$ and $\mathbb{E} Z_{0, j}^{\leq}=-\mathbb{E} Z_{0, j}^{>}$. Conditioning as before, we conclude

$$
\mathbb{E} Z_{0, j}^{\leq}=-\mathbb{E} Z_{0, j}^{>}=-\mathbb{E} Y_{1, n}^{>} \mathbb{E} N_{1}^{>} \int_{j}^{j+1} f(-s) \mathrm{d} s .
$$

By Bingham et al. (1989, Theorem 1.6.5)

$$
-\lim _{n \rightarrow \infty} \frac{n \mathbb{E} N_{1}^{>} \mathbb{E} Y_{1, n}^{>}}{a_{n}}=\lim _{n \rightarrow \infty} \frac{n}{a_{n}} \int_{x>a_{n}} x \bar{\nu}(\mathrm{d} x)=\frac{\alpha(p-q)}{(1-\alpha)} .
$$


For $\alpha=1$ we assumed symmetry and therefore the centering vanishes and hence we proved (4.17) for all $\alpha \in(0,2)$. With the same arguments the convergence

$$
\frac{1}{a_{n}}\left(\sum_{t=1}^{n} X_{t}^{+}-n \sum_{j=-\infty}^{\infty} \mathbb{E} Z_{0, j}^{\leq}\right) \stackrel{d}{\rightarrow} S_{\alpha}\left(\beta_{1}, \gamma_{1}, \delta_{1}\right) \quad(n \rightarrow \infty),
$$

where $\delta_{1}=-\frac{\alpha(p-q)}{1-\alpha} \int_{-\infty}^{\infty} f(-s) \mathrm{d} s$, is equivalent to $a_{n}^{-1} \sum_{t=1}^{n} X_{t}^{+} \stackrel{d}{\rightarrow} S_{\alpha}\left(\beta_{1}, \gamma_{1}, 0\right)$ and (4.19) follows by Billingsley (1968, Theorem 4.2) if we can show that the following two conditions are satisfied

$$
\begin{array}{r}
S_{\alpha}\left(\beta_{1, m}, \gamma_{1, m}, \delta_{1, m}\right) \stackrel{w}{\rightarrow} S_{\alpha}\left(\beta_{1}, \gamma_{1}, \delta_{1}\right) \quad(m \rightarrow \infty),( \\
\lim _{m \rightarrow \infty} \limsup _{n \rightarrow \infty} \mathbb{P}\left(a_{n}^{-1}\left|\sum_{t=1}^{n}\left(X_{t}^{+}-X_{t, m}^{+}\right)-n \sum_{j>|m|, j=m} \mathbb{E} Z_{0, j}^{\leq}\right|>\epsilon\right)=0 \quad \forall \epsilon>0 .
\end{array}
$$

By assumption $f \in L^{1}(\mathbb{R})$ and hence $\delta_{1, m} \rightarrow \delta_{1}$ as $m \rightarrow \infty$. Since we have shown before that $c_{1}^{ \pm}=\lim _{m \rightarrow \infty} c_{1, m}^{ \pm}$it holds that $\beta_{1, m} \rightarrow \beta_{1}$ and $\gamma_{1, m} \rightarrow \gamma_{1}$. Observe that the characteristic function of the distribution $S_{\alpha}(\beta, \gamma, \delta)$ is continuous in $\beta, \gamma$ and $\delta$, and therefore the first condition (4.20) follows. Notice that

$$
\sum_{t=1}^{n}\left(X_{t}^{+}-X_{t, m}^{+}\right)-n \sum_{\substack{j>|m|, j=m}} \mathbb{E} Z_{0, j}^{\leq}=\sum_{t=1}^{n} \sum_{k \in\{-\infty, \ldots, t-m-1\} \cup\{t+m, \ldots, \infty\}}\left(\widetilde{Z}_{t, k}^{\leq}+Z_{t, k}^{>}\right) .
$$

Rearranging yields

$\sum_{t=1}^{n} \sum_{k \in\{-\infty, \ldots, t-m-1\} \cup\{t+m, \ldots, \infty\}} \widetilde{Z}_{t, k}^{\leq}=\sum_{j=m+1}^{\infty} \sum_{i=1}^{n \wedge(j-m)} \widetilde{Z}_{i, j}^{\leq}+\sum_{j=-\infty}^{-m-1+n} \sum_{i=1 \vee(m+1+j)}^{n} \widetilde{Z}_{i, j}^{\leq}$.

Hence we can bound the probability in (4.21) by

$$
\begin{aligned}
\mathbb{P}\left(a_{n}^{-1}\left|\sum_{j=m+1}^{\infty} \sum_{i=1}^{n \wedge(j-m)} \widetilde{Z}_{i, j}^{\leq}+\sum_{j=-\infty}^{-m-1+n} \sum_{i=1 \vee(m+1+j)}^{n} \widetilde{Z}_{i, j}^{\leq}\right|>\frac{\epsilon}{2}\right) \\
\quad+\mathbb{P}\left(a_{n}^{-1}\left|\sum_{t=1}^{n}\left(\sum_{k=-\infty}^{t-m-1}+\sum_{k=t+m}^{\infty}\right) Z_{t, k}^{>}\right|>\frac{\epsilon}{2}\right) .
\end{aligned}
$$

We first show that the $\lim _{m \rightarrow \infty} \limsup _{n \rightarrow \infty}$ of the first term is zero. Observe that $\operatorname{Var}(A+B) \leq 2(\operatorname{Var}(A)+\operatorname{Var}(B))$ for arbitrary random variables $A$ and $B$. Hence applying the Chebyshev inequality yields

$$
\begin{array}{r}
\mathbb{P}\left(a_{n}^{-1}\left|\sum_{j=m+1}^{\infty} \sum_{i=1}^{n \wedge(j-m)} \widetilde{Z}_{i, j}^{\leq}+\sum_{j=-\infty}^{-m-1+n} \sum_{i=1 \vee(m+1+j)}^{n} \widetilde{Z}_{i, j}^{\leq}\right|>\epsilon / 2\right) \\
\leq \frac{8}{a_{n}^{2} \epsilon^{2}}\left(\sum_{j=m+1}^{\infty} \operatorname{Var}\left(\sum_{i=1}^{n \wedge(j-m)} Z_{i, j}^{\leq}\right)+\sum_{j=-\infty}^{-m-1+n} \operatorname{Var}\left(\sum_{i=1 \vee(m+1+j)}^{n} Z_{i, j}^{\leq}\right)\right) .
\end{array}
$$

Denote the quadratic variation of a semimartingale $M=\left(M_{s}\right)_{s \geq 0}$ on the interval $[0, s]$ by $[M, M]_{s}$ and notice that $L^{\leq}=\left(L_{S}^{\leq}\right)_{s \geq 0}$ with the compound Poisson representation from (L2) has quadratic variation $\left[L^{\leq}, L^{\leq}\right]_{s}=\sum_{k=1}^{N_{s}^{\leq}}\left(Y_{k, n}^{\leq}\right)^{2}$ for $s \in[0,1]$. 
Hence by the Itô-isometry we conclude for $g(s):=\sum_{i=1}^{n \wedge(j-m)} f(i-j-s)$

$$
\begin{aligned}
\operatorname{Var}\left(\sum_{i=1}^{n \wedge(j-m)} Z_{i, j}^{\leq}\right) & =\operatorname{Var}\left(\int_{0}^{1}\left(\sum_{i=1}^{n \wedge(j-m)} f(i-j-s)\right) \mathrm{d} L_{s}^{\leq}\right) \\
& =\mathbb{E}\left[\left(\int_{0}^{1} g(s) \mathrm{d} L_{s}^{\leq}\right)^{2}\right]-\left(\mathbb{E} \int_{0}^{1} g(s) \mathrm{d} L_{s}^{\leq}\right)^{2} \\
& =\mathbb{E} \int_{0}^{1} g^{2}(s) \mathrm{d}\left[L^{\leq}, L^{\leq}\right]_{s}=\mathbb{E}\left[\left(Y_{1, n}^{\leq}\right)^{2}\right] \mathbb{E} N_{1}^{\leq} \int_{0}^{1} g^{2}(s) \mathrm{d} s .
\end{aligned}
$$

Thus we get

$$
\begin{aligned}
& \frac{1}{a_{n}^{2}} \sum_{j=m+1}^{\infty} \operatorname{Var}\left(\sum_{i=1}^{n \wedge(j-m)} Z_{i, j}^{\leq}\right)= \\
& =\left(\frac{1}{a_{n}^{2}} \mathbb{E} N_{1}^{\leq} \mathbb{E}\left[\left(Y_{1, n}^{\leq}\right)^{2}\right]\right) \sum_{j=m+1}^{\infty} \int_{0}^{1}\left(\sum_{i=1}^{n \wedge(j-m)} f(i-j-s)\right)^{2} \mathrm{~d} s \\
& =\frac{\mathbb{E} N_{1}^{\leq} \mathbb{E}\left[\left(Y_{1, n}^{\leq}\right)^{2}\right]}{a_{n}^{2}}\left(\sum_{j=m+1}^{n+m} \int_{0}^{1}\left(\sum_{i=1-j}^{-m} f(i-s)\right)^{2} \mathrm{~d} s+\sum_{j=n+m+1}^{\infty} \int_{0}^{1}\left(\sum_{i=1-j}^{n-j} f(i-s)\right)^{2} \mathrm{~d} s\right)
\end{aligned}
$$

By Bingham et al. (1989, Theorem 1.6.4) it holds that

$$
\limsup _{n \rightarrow \infty} \frac{n}{a_{n}^{2}} \mathbb{E} N_{1}^{\leq} \mathbb{E}\left[\left(Y_{1, n}^{\leq}\right)^{2}\right]=\limsup _{n \rightarrow \infty} \frac{n}{a_{n}^{2}} \int_{1<|x| \leq a_{n}}|x|^{2} \nu(\mathrm{d} x)<\infty .
$$

The first sum in (4.24) satisfies since $\bar{F}_{1} \in L^{2}([0,1])$, as $n \rightarrow \infty$,

$$
\begin{aligned}
\frac{1}{n} \sum_{j=m+1}^{n+m} \int_{0}^{1}\left(\sum_{i=1-j}^{-m} f(i-s)\right)^{2} \mathrm{~d} s & =\frac{1}{n} \sum_{j=1}^{n} \int_{0}^{1}\left(\sum_{i=1-j-m}^{-m} f(i-s)\right)^{2} \mathrm{~d} s \\
& \rightarrow \int_{0}^{1}\left(\sum_{i=-\infty}^{-m} f(i-s)\right)^{2} \mathrm{~d} s .
\end{aligned}
$$

Similarly, we calculate for the second term in (4.24)

$$
\begin{aligned}
\frac{1}{n} \sum_{j=n+m+1}^{\infty} \int_{0}^{1}\left(\sum_{i=1-j}^{n-j} f(i-s)\right)^{2} \mathrm{~d} s & =\frac{1}{n} \sum_{j=m+1}^{\infty} \int_{0}^{1}\left(\sum_{i=1-j-n}^{-j} f(i-s)\right)^{2} \mathrm{~d} s \\
& \leq \int_{0}^{1}\left(\sum_{i=-\infty}^{-m-1}|f(i-s)|\right)^{2} \mathrm{~d} s
\end{aligned}
$$

Hence $\lim _{m \rightarrow \infty} \lim \sup _{n \rightarrow \infty} a_{n}^{-2} \sum_{j=m+1}^{\infty} \operatorname{Var}\left(\sum_{i=1}^{n \wedge(j-m)} Z_{i, j}^{\leq}\right)=0$ by equations (4.24)-(4.25), and a similar argument applies to the second term in (4.23). Suppose $\alpha \in(1,2)$. The second term in (4.22) can be bounded from above by

$$
\mathbb{P}\left(a_{n}^{-1}\left|\sum_{t=1}^{n}\left(\sum_{k=-\infty}^{t-m-1}+\sum_{k=t+m}^{\infty}\right) Z_{t, k}^{>}\right|>\epsilon / 2\right) \leq \frac{2}{\epsilon a_{n}} \sum_{t=1}^{n}\left(\sum_{k=-\infty}^{t-m-1}+\sum_{k=t+m}^{\infty}\right) \mathbb{E}\left|Z_{t, k}^{>}\right| .
$$


As before in (4.18), by conditioning on the number of jumps and jump times and the triangle inequality we conclude

$$
\mathbb{E}\left|Z_{t, k}^{>}\right| \leq \mathbb{E}\left|Y_{1, n}^{>}\right| \mathbb{E} \int_{0}^{1}|f(t-k-s)| \mathrm{d} N_{s}^{>}=\mathbb{E}\left|Y_{1, n}^{>}\right| \mathbb{E} N_{1}^{>} \int_{0}^{1}|f(t-k-s)| \mathrm{d} s .
$$

Applying again Bingham et al. (1989, Theorem 1.6.5) yields

$$
\limsup _{n \rightarrow \infty} \frac{n \mathbb{E}\left|Y_{1, n}^{>}\right| \mathbb{E} N_{1}^{>}}{a_{n}}=\limsup _{n \rightarrow \infty} \frac{n}{a_{n}} \int_{|x| \geq a_{n}}|x| \nu(\mathrm{d} x)<\infty .
$$

Further, we have

$$
\frac{1}{n} \sum_{t=1}^{n}\left(\sum_{k=-\infty}^{t-m-1}+\sum_{k=t+m}^{\infty}\right) \int_{0}^{1}|f(t-k-s)| \mathrm{d} s=\int_{-\infty}^{-m}|f(-s)| \mathrm{d} s+\int_{m}^{\infty}|f(-s)| \mathrm{d} s .
$$

Hence

$$
\mathbb{P}\left(a_{n}^{-1}\left|\sum_{t=1}^{n}\left(\sum_{k=-\infty}^{t-m-1}+\sum_{k=t+m}^{\infty}\right) Z_{t, k}^{>}\right|>\frac{\epsilon}{2}\right) \leq \frac{2}{\epsilon} \frac{n}{a_{n}} \mathbb{E}\left|Y_{1, n}^{>}\right| \mathbb{E} N_{1}^{>} \int_{-\infty}^{\infty}|f(-s)| \mathrm{d} s
$$

and since $f \in L^{1}(\mathbb{R})$ the $\lim _{m \rightarrow \infty} \lim \sup _{n \rightarrow \infty}$ of this term is zero. If $\alpha \in(0,1]$, choose $\delta<\alpha, \delta \leq 1$ such that $f \in L^{\delta}(\mathbb{R})$. Then by Chebyshev's inequality and the subadditivity of $x \mapsto|x|^{\delta}, \delta \leq 1$,

$\mathbb{P}\left(a_{n}^{-1}\left|\sum_{t=1}^{n}\left(\sum_{k=-\infty}^{t-m-1}+\sum_{k=t+m}^{\infty}\right) Z_{t, k}^{>}\right|>\epsilon / 2\right) \leq \frac{2^{\delta}}{\epsilon^{\delta} a_{n}^{\delta}} \sum_{t=1}^{n}\left(\sum_{k=-\infty}^{t-m-1}+\sum_{k=t+m}^{\infty}\right) \mathbb{E}\left|Z_{t, k}^{>}\right|^{\delta}$.

Conditioning as before gives

$$
\mathbb{E}\left|Z_{t, k}^{>}\right|^{\delta} \leq \mathbb{E}\left|Y_{1, n}^{>}\right|^{\delta} \mathbb{E} \int_{0}^{1}|f(t-k-s)|^{\delta} \mathrm{d} N_{s}^{>}=\mathbb{E}\left|Y_{1, n}^{>}\right|^{\delta} \mathbb{E} N_{1}^{>} \int_{0}^{1}|f(t-k-s)|^{\delta} \mathrm{d} s,
$$

and applying again Bingham et al. (1989, Theorem 1.6.5) yields

$$
\limsup _{n \rightarrow \infty} \frac{n \mathbb{E}\left|Y_{1, n}^{>}\right|^{\delta} \mathbb{E} N_{1}^{>}}{a_{n}^{\delta}}=\limsup _{n \rightarrow \infty} \frac{n}{a_{n}^{\delta}} \int_{|x| \geq a_{n}}|x|^{\delta} \nu(\mathrm{d} x)<\infty .
$$

Similar calculations as before complete the proof of (4.21) and hence the claim is proved when $c_{1}^{+}+c_{1}^{-}>0$. If $c_{1}^{+}+c_{1}^{-}=0$, then there is either an increasing subsequence $\left(m_{i}\right)_{i \in \mathbb{N}} \subset \mathbb{N}$, i.e. $m_{i} \leq m_{i+1}$ for all $i \in \mathbb{N}$, such that $c_{1, m_{i}}^{+}+c_{1, m_{i}}^{-}=0$ for all $i \in \mathbb{N}$, or one such that $c_{1, m_{i}}^{+}+c_{1, m_{i}}^{-}>0$ for all $i \in \mathbb{N}$. In the first case Proposition 2 implies $a_{n}^{-1} \sum_{t=1}^{n} X_{t, m_{i}}^{+} \stackrel{\mathbb{P}}{\rightarrow} 0$ as $n \rightarrow \infty$ for every $i \in \mathbb{N}$ and the statement of the theorem follows in this case also from (4.21), which has been already proved. In the second case, (4.16) holds along $\left(m_{i}\right)_{i \in \mathbb{N}}$ and $\gamma_{1, m_{i}}^{\alpha} \rightarrow 0$ as well $\gamma_{1, m_{i}}^{\alpha} \beta_{1, m_{i}} \rightarrow 0$ as $i \rightarrow \infty$. Hence $S_{\alpha / 2}\left(\beta_{1, m_{i}}, \gamma_{1, m_{i}}, 0\right) \stackrel{w}{\rightarrow} \delta_{0}$ as $i \rightarrow \infty$ by (3.1), where $\delta_{0}$ denotes the Dirac measure at 0 .

Assume (A1) and (A2) are satisfied and define for fixed sampling frequency $\Delta>0, h \geq 0$ and $Y_{t, h}=X_{t} X_{t+h}$ with $\left(X_{t}\right)_{t \in \mathbb{R}}$ in (2.5) the sample autocovariance with lag $h$ by

$$
\gamma_{n, \Delta}(h)=n^{-1} \sum_{t=1}^{n} X_{\Delta t} X_{\Delta(t+h)}=n^{-1} \sum_{t=1}^{n} Y_{\Delta t, \Delta h}, \quad n \in \mathbb{N} .
$$


Further, denote for $m \in \mathbb{N}$ and $f_{\Delta m}(s)=f(s) \mathbb{1}_{[-m \Delta, m \Delta]}(s)$ the random variables

$$
\begin{aligned}
X_{t, \Delta m} & :=\int_{-\infty}^{\infty} f_{\Delta m}(t-s) \mathrm{d} L_{s}=\int_{t-m \Delta}^{t+m \Delta} f(t-s) \mathrm{d} L_{s}, \quad t \in \mathbb{R}, \\
Y_{t, h, \Delta, m} & :=X_{\Delta t, \Delta m} X_{\Delta(t+h), \Delta m} .
\end{aligned}
$$

By Proposition 1(ii), $Y_{t, h} \in R V\left(\alpha / 2, a_{n}^{2}, \mu_{f_{1+h}} \circ g_{1, h}^{-1}\right)$ for $h \in \mathbb{N}_{0}$. Hence one concludes by the same arguments as in the proof of Proposition 9 that for every fixed $h \in \mathbb{N}_{0}$ the sequence $\left(Y_{t, h}\right)_{t \in \mathbb{Z}}$ is regularly varying in the sense of equations (4.3) and (4.4) with $r_{n}=\left(\int_{-\infty}^{\infty}|f(s) f(s+h)|^{\alpha / 2} \mathrm{~d} s\right)^{2 / \alpha} a_{n}^{2}$ and $\mu_{d}:=\left(\mu_{f_{d+h}} \circ\right.$ $\left.g_{d, h}^{-1}\right) /\left(\int_{-\infty}^{\infty}|f(s) f(s+h)|^{\alpha / 2} \mathrm{~d} s\right)$.

Proposition 4. Let $\Delta>0, h \in \mathbb{N}_{0}$ and $m \in \mathbb{N}$ sufficiently large, so that $h<2 m$ and assume that $\int_{-\infty}^{\infty}\left|f_{\Delta m}(s) f_{\Delta m}(s+\Delta h)\right| \mathrm{d} s>0$. Assume that (A1) and (AV) are satisfied and let $\left(Y_{t, h, \Delta, m}\right)_{t \in \mathbb{Z}}$ be defined as in (4.27). For $S_{n, h, \Delta, m}=\sum_{t=1}^{n} Y_{t, h, \Delta, m}$ the limits

$$
b_{h, \Delta, m}^{+}(d)=\lim _{n \rightarrow \infty} \mathbb{P}\left(S_{d, h, \Delta, m}>a_{n}^{2}\right), b_{h, \Delta, m}^{-}(d)=\lim _{n \rightarrow \infty} \mathbb{P}\left(S_{d, h, \Delta, m}<-a_{n}^{2}\right), \quad d \geq 1,
$$

exist and the parameters $c_{h, \Delta, m}^{ \pm}=b_{h, \Delta, m}^{ \pm}(2 m+h)-b_{h, \Delta, m}^{ \pm}(2 m+h-1)$ are given for $F_{h, \Delta, m}(s)=\sum_{j=1-m}^{m-h} f(\Delta j-s) f(\Delta(j+h)-s)$ by

$$
c_{h, \Delta, m}^{+}=\int_{0}^{\Delta}\left(F_{h, \Delta, m}^{+}(s)\right)^{\alpha / 2} \mathrm{~d} s, \quad c_{h, \Delta, m}^{-}=\int_{0}^{\Delta}\left(F_{h, \Delta, m}^{-}(s)\right)^{\alpha / 2} \mathrm{~d} s .
$$

(i) If $c_{h, \Delta, m}^{+}+c_{h, \Delta, m}^{-}>0$, then $a_{n}^{-2} \sum_{t=1}^{n} Y_{t, h, \Delta, m} \stackrel{d}{\rightarrow} S_{\alpha / 2}\left(\beta_{h, \Delta, m}, \gamma_{h, \Delta, m}, 0\right)$, where the parameters $\beta_{h, \Delta, m}, \gamma_{h, \Delta, m}$ are given by

$$
\beta_{h, \Delta, m}=\frac{c_{h, \Delta, m}^{+}-c_{h, \Delta, m}^{-}}{c_{h, \Delta, m}^{+}+c_{h, \Delta, m}^{-}}, \quad \gamma_{h, \Delta, m}^{\alpha / 2}=\left(c_{h, \Delta, m}^{+}+c_{h, \Delta, m}^{-}\right) \frac{\Gamma\left(2-\frac{\alpha}{2}\right)}{1-\frac{\alpha}{2}} \cos \left(\frac{\pi \alpha}{4}\right) .(4
$$

(ii) If $c_{h, \Delta, m}^{+}+c_{h, \Delta, m}^{-}=0$, then $a_{n}^{-2} \sum_{t=1}^{n} Y_{t, h, \Delta, m} \stackrel{\mathbb{P}}{\rightarrow} 0$.

Proof: Observe that the sequence $\left(Y_{t, h, \Delta, m}\right)_{t \in \mathbb{Z}}$ is $(2 m+h-1)$-dependent. The proof of part (i) and (ii) is completely analogue to the proof of Proposition 3 and therefore omitted. It remains to show (4.28). To this end, assume w.l.o.g. $\Delta=1$. With the notation as in the proof of Proposition 3 and by Proposition 1(ii) we get

$$
\begin{aligned}
b_{h, 1, m}^{+}(2 m+h) & =\left(\mu_{f_{2 m+2 h}^{(m)}} \circ g_{2 m+h, h}^{-1}\right)\left(\varphi^{-1}((1, \infty))\right) \\
& =\int_{-\infty}^{\infty}\left(\left(\sum_{i=1}^{2 m+h} f_{m}(i-s) f_{m}(i+h-s)\right)^{+}\right)^{\alpha / 2} \mathrm{~d} s .
\end{aligned}
$$

Notice that $f_{m}(i-s) f_{m}(i+h-s)=0$ if $s \notin[i+h-m, i+m]$. Hence

$$
b_{h, 1, m}^{+}(2 m+h)=\sum_{j=1-m+h}^{3 m+h-1} \int_{j}^{j+1}\left(\left(\sum_{i=1 \vee(j-m+1)}^{(2 m+h) \wedge(m-h+j)} f(i-s) f(i+h-s)\right)^{+}\right)^{\alpha / 2} \mathrm{~d} s .
$$


Further, for $w(z):=f(z) f(z+h), z \in \mathbb{R}$, we get by index changes

$$
\begin{aligned}
& b_{h, 1, m}^{+}(2 m+h)=\sum_{j=1-m+h}^{3 m+h-1} \int_{0}^{1}\left(\left(\sum_{i=(1-j) \vee(-m+1)}^{(2 m+h-j) \wedge(m-h)} w(i-s)\right)^{+}\right)^{\frac{\alpha}{2}} \mathrm{~d} s \\
& =\sum_{j=1-m+h}^{m} \int_{0}^{1}\left(\left(\sum_{i=1-j}^{m-h} w(i-s)\right)^{+}\right)^{\frac{\alpha}{2}} \mathrm{~d} s+\sum_{j=m+1}^{m+2 h-1} \int_{0}^{1}\left(\left(\sum_{i=-m+1}^{m-h} w(i-s)\right)^{+}\right)^{\frac{\alpha}{2}} \mathrm{~d} s \\
& +\sum_{j=m+2 h}^{3 m+h-1} \int_{0}^{1}\left(\left(\sum_{i=-m+1}^{2 m+h-j} w(i-s)\right)^{+}\right)^{\frac{\alpha}{2}} \mathrm{~d} s .
\end{aligned}
$$

Similarly, one deduces

$$
\begin{aligned}
& b_{h, 1, m}^{+}(2 m+h-1)=\sum_{j=1-m+h}^{m} \int_{0}^{1}\left(\left(\sum_{i=1-j}^{m-h} w(i-s)\right)^{+}\right)^{\frac{\alpha}{2}} \mathrm{~d} s \\
& +\sum_{j=m+1}^{m+2 h-1} \int_{0}^{1}\left(\left(\sum_{i=-m+1}^{m-h} w(i-s)\right)^{+}\right)^{\frac{\alpha}{2}} \mathrm{~d} s+\sum_{j=m+2 h}^{3 m+h-2} \int_{0}^{1}\left(\left(\sum_{i=-m+1}^{2 m+h-j-1} w(i-s)\right)^{+}\right)^{\frac{\alpha}{2}} \mathrm{~d} s .
\end{aligned}
$$

Hence

$$
\begin{aligned}
c_{h, 1, m}^{+} & =b_{h, 1, m}^{+}(2 m+h)-b_{h, 1, m}^{+}(2 m+h-1) \\
& =\int_{0}^{1}\left(\left(\sum_{i=1-m}^{m-h} f(i-s) f(i+h-s)\right)^{+}\right)^{\alpha / 2} \mathrm{~d} s
\end{aligned}
$$

and the same calculations for $c_{h, 1, m}^{-}$complete the proof.

Proof of Theorem 3. W.l.o.g. we assume $\Delta=1$ (other cases follow from a time change, see (4.1)). Applying the Lévy-Itô decomposition (L1), we can write $L_{t}=$ $L_{t}^{+}+L_{t}^{-}$for $t \in \mathbb{R}$ and define $X_{t}^{ \pm}=\int_{-\infty}^{\infty} f(t-s) \mathrm{d} L_{s}^{ \pm}$. Let $\epsilon>0$ and $\tau \in(\alpha / 2,1)$. Then by the subadditivity of $x \mapsto|x|^{\tau}$ and (2.4), as $n \rightarrow \infty$,

$$
\mathbb{P}\left(\left|\sum_{t=1}^{n} X_{t}^{+} X_{t+h}^{-}\right|>\epsilon a_{n}^{2}\right) \leq \frac{\mathbb{E}\left|\sum_{t=1}^{n} X_{t}^{+} X_{t+h}^{-}\right|^{\tau}}{\epsilon^{\tau} a_{n}^{2 \tau}} \leq \epsilon^{-\tau} n a_{n}^{-2 \tau} \mathbb{E}\left|X_{0}^{+}\right|^{\tau} \mathbb{E}\left|X_{0}^{-}\right|^{\tau} \rightarrow 0 .
$$

A similar argument applies for $\sum_{t=1}^{n} X_{t}^{-} X_{t+h}^{+}$and $\sum_{t=1}^{n} X_{t}^{-} X_{t+h}^{-}$, so that

$$
a_{n}^{-2} \sum_{t=1}^{n}\left(X_{t}^{+} X_{t+h}^{-}+X_{t}^{-} X_{t+h}^{+}+X_{t}^{-} X_{t+h}^{-}\right) \stackrel{\mathbb{P}}{\rightarrow} 0 \quad(n \rightarrow \infty)
$$

and hence by Slutsky's theorem

$$
d-\lim _{n \rightarrow \infty} a_{n}^{-2} \sum_{t=1}^{n} X_{t} X_{t+h}=d-\lim _{n \rightarrow \infty} a_{n}^{-2} \sum_{t=1}^{n} X_{t}^{+} X_{t+h}^{+} .
$$

Since $G_{0,1} \in L^{\alpha / 2}([0,1])$ we also have $u \mapsto \sum_{j \in \mathbb{Z}}|f(j-u) f(j+h-u)| \in L^{\alpha / 2}([0,1])$ by Hölder's inequality, and hence by the Theorem of Lebesgue the limits $\lim _{m \rightarrow \infty} c_{h, 1, m}^{ \pm}$of $c_{h, 1, m}^{ \pm}$in (4.28) exist and are equal to $c_{h, 1}^{ \pm}$. Suppose first that $c_{h, 1}^{+}+c_{h, 1}^{-}>0$, so that also $c_{h, 1, m}^{+}+c_{h, 1, m}^{-}>0$ for large $m$. Since $\int_{\mathbb{R}}|f(s) f(s+h)| \mathrm{d} s>$ 0 , also $\int_{\mathbb{R}}\left|f_{m}(s) f_{m}(s+h)\right| \mathrm{d} s>0$ for large $m \in \mathbb{N}$ and we have by Proposition 4 for $X_{t, m}^{+}=\int_{-\infty}^{\infty} f_{m}(t-s) \mathrm{d} L_{s}^{+}$that $a_{n}^{-2} \sum_{t=1}^{n} X_{t, m}^{+} X_{t+h, m}^{+} \stackrel{d}{\rightarrow} S_{\alpha / 2}\left(\beta_{h, 1, m}, \gamma_{h, 1, m}, 0\right)$ 
as $n \rightarrow \infty$, where the parameters are given in equation (4.29). Hence it suffices by Theorem 4.2 (Billingsley, 1968) to show that

$$
\begin{array}{r}
S_{\alpha / 2}\left(\beta_{h, 1, m}, \gamma_{h, 1, m}, 0\right) \stackrel{w}{\rightarrow} S_{\alpha / 2}\left(\beta_{h, 1}, \gamma_{h, 1}, 0\right) \quad(m \rightarrow \infty), \\
\lim _{m \rightarrow \infty} \limsup _{n \rightarrow \infty} \mathbb{P}\left(a_{n}^{-2}\left|\sum_{t=1}^{n}\left(X_{t}^{+} X_{t+h}^{+}-X_{t, m}^{+} X_{t+h, m}^{+}\right)\right|>\epsilon\right)=0 \quad \forall \epsilon>0 .
\end{array}
$$

But $\beta_{h, 1, m} \rightarrow \beta_{h, 1}$ and $\gamma_{h, 1, m} \rightarrow \gamma_{h, 1}$ as $m \rightarrow \infty$ and hence the first statement (4.30) follows from the continuity of the characteristic function of the stable distribution. Denote $Z_{t, i}^{+}=\int_{i}^{i+1} f(t-s) \mathrm{d} L_{s}^{+}$for $t \in \mathbb{R}$ and $i \in \mathbb{Z}$ and let $\delta<\alpha, \delta \leq 1$ as in (A2). Notice that $\sum_{k \in \mathbb{Z}}\left|Z_{t, k}^{+}\right|$converges almost surely absolutely since by the subadditivity of $x \mapsto|x|^{\delta}$ and by applying the compound Poisson representation from (L1) and conditioning on the number of jumps and jump times as in (4.18),

$$
\left.\mathbb{E}\left|\sum_{k \in \mathbb{Z}}\right| Z_{t, k}^{+}\right|^{\delta} \leq \sum_{k \in \mathbb{Z}} \mathbb{E}\left|Z_{t, k}^{+}\right|^{\delta} \leq\left(|\gamma|^{\delta}+\mathbb{E} N_{1}^{+} \mathbb{E}\left|Y_{1}^{+}\right|^{\delta}\right) \int_{\mathbb{R}}|f(s)|^{\delta} \mathrm{d} s<\infty
$$

Observe that

$$
X_{t}^{+} X_{t+h}^{+}-X_{t, m}^{+} X_{t+h, m}^{+}=\sum_{\substack { k \in \mathbb{Z} \\
\begin{subarray}{c}{j-t-h \mid>m, j=t+h+m{ k \in \mathbb { Z } \\
\begin{subarray} { c } { j - t - h | > m , \\
j = t + h + m } }\end{subarray}} Z_{t, k}^{+} Z_{t+h, j}^{+}+\sum_{\substack{|k-t|>m, j=t+h-m \\
k=t+m}} \sum_{t, k}^{t+h+m-1} Z_{t+h, j}^{+} Z^{+}
$$

Applying the Lévy-Itô decomposition (L2), we define for $t \in \mathbb{R}$ and $k \in \mathbb{Z}$

$$
Z_{t, k}^{\leq}:=\int_{k}^{k+1} f(t-s) \mathrm{d} L_{s}^{\leq}, \quad Z_{t, k}^{>}:=\int_{k}^{k+1} f(t-s) \mathrm{d} L_{s}^{>} .
$$

By similar estimates as in (4.32), $\mathbb{E}\left|\sum_{k \in \mathbb{Z}}\right| Z_{t, k}^{\leq}||^{\delta}<\infty$ and $\mathbb{E}\left|\sum_{k \in \mathbb{Z}}\right| Z_{t, k}^{>}||^{\delta}<\infty$. Hence $\sum_{k \in \mathbb{Z}} Z_{t, k}^{\leq}$and $\sum_{k \in \mathbb{Z}} Z_{t, k}^{>}$are also almost surely absolutely convergent.

Notice that $\mathbb{P}\left(a_{n}^{-2}\left|\sum_{t=1}^{n}\left(X_{t}^{+} X_{t+h}^{+}-X_{t, m}^{+} X_{t+h, m}^{+}\right)\right|>\epsilon\right)$ can be bounded from above by

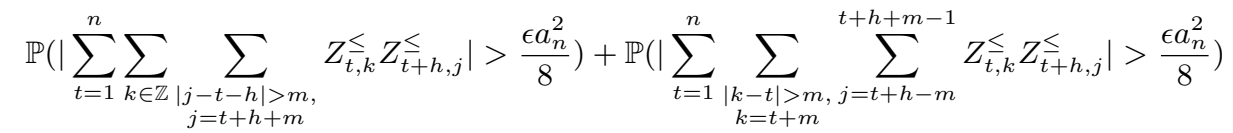

$$
\begin{aligned}
& +\mathbb{P}\left(\left|\sum_{t=1}^{n} \sum_{k \in \mathbb{Z}} \sum_{\substack{j-t-h \mid>m, j=t+h+m}} Z_{t, k}^{>} Z_{t+h, j}^{>}\right|>\frac{\epsilon a_{n}^{2}}{8}\right)+\mathbb{P}\left(\left|\sum_{t=1}^{n} \sum_{\substack{k-t \mid>m, j=t+h-m \\
k=t+m}}^{t+h+m-1} \sum_{t, k}^{>} Z_{t+h, j}^{>}\right|>\frac{\epsilon a_{n}^{2}}{8}\right)
\end{aligned}
$$

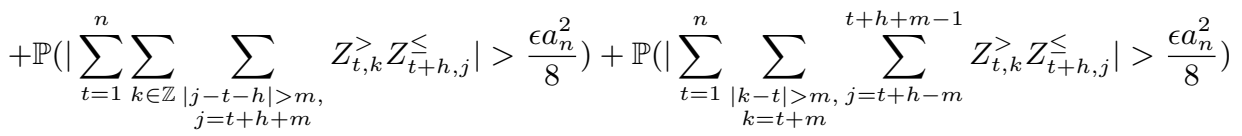

$$
\begin{aligned}
& +\mathbb{P}\left(\left|\sum_{t=1}^{n} \sum_{k \in \mathbb{Z}} \sum_{\substack{j-t-h \mid>m, j=t+h+m}} Z_{t, k}^{\leq} Z_{t+h, j}^{>}\right|>\frac{\epsilon a_{n}^{2}}{8}\right)+\mathbb{P}\left(\left|\sum_{t=1}^{n} \sum_{\substack{k-t \mid>m, j \\
k=t+m}} \sum_{j=t+h-m}^{t+h+m-1} Z_{t, k}^{\leq} Z_{t+h, j}^{>}\right|>\frac{\epsilon a_{n}^{2}}{8}\right) .
\end{aligned}
$$


We consider first the third term in (4.33). Let $\eta=\delta / 2$. Then by Chebyshev's inequality

$$
\begin{aligned}
& \mathbb{P}\left(a_{n}^{-2}\left|\sum_{t=1}^{n} \sum_{k \in \mathbb{Z}} \sum_{\substack{|j-t-h|>m, j=t+h+m}} Z_{t, k}^{>} Z_{t+h, j}^{>}\right|>\frac{\epsilon}{8}\right) \leq \frac{16^{\eta}}{\epsilon^{\eta} a_{n}^{2 \eta}} \sum_{t=1}^{n} \sum_{\substack{|j-t-h|>m, j=t+h+m}} \mathbb{E}\left|Z_{t, j}^{>} Z_{t+h, j}^{>}\right|^{\eta} \\
& +\frac{16^{2 \eta}}{\epsilon^{2 \eta} a_{n}^{4 \eta}} \sum_{t=1}^{n} \sum_{\substack{|j-t-h|>m \\
j=t+h+m}} \sum_{k \in \mathbb{Z}, k \neq j} \mathbb{E}\left|Z_{t, k}^{>}\right|^{2 \eta} \mathbb{E}\left|Z_{t+h, j}^{>}\right|^{2 \eta}
\end{aligned}
$$

Applying the compound Poisson representation from (L2), the Cauchy-Schwarz inequality and the subadditivity of $x \mapsto|x|^{\eta}$ we conclude by conditioning on the number of jumps and jump times of $\left(N_{s}^{>}\right)_{s \in[0,1]}$

$$
\begin{aligned}
\mathbb{E}\left|Z_{t, j}^{>} Z_{t+h, j}^{>}\right|^{\eta} & =\mathbb{E}\left|\int_{0}^{1} f(t-j-s) \mathrm{d} L_{s}^{>} \int_{0}^{1} f(t+h-j-s) \mathrm{d} L_{s}^{>}\right|^{\eta} \\
& =\mathbb{E}\left|\left(\sum_{i=1}^{N_{s}^{>}} f\left(t-j-T_{i}^{>}\right) Y_{i, n}^{>}\right)\left(\sum_{l=1}^{N_{s}^{>}} f\left(t+h-j-T_{l}^{>}\right) Y_{l, n}^{>}\right)\right|^{\eta} \\
& \leq \mathbb{E}\left|Y_{1, n}^{>}\right|^{2 \eta} \mathbb{E}\left(\int_{0}^{1} \|\left. f(t-j-s)\right|^{\eta} \mathrm{d} N_{s}^{>} \int_{0}^{1}|f(t+h-j-s)|^{\eta} \mathrm{d} N_{s}^{>}\right)
\end{aligned}
$$

Denote $g_{t}(s):=|f(t-s)|^{\eta}$ for $t, s \in \mathbb{R}$ and $\widetilde{N}_{s}^{>}:=N_{s}^{>}-s \mathbb{E} N_{1}^{>}$for $s \in[0,1]$. By the Itô-isometry and since the quadratic variation of a Poisson process is the process itself, it follows that

$$
\begin{aligned}
& \mathbb{E}\left(\int_{0}^{1} g_{t-j}(s) \mathrm{d} N_{s}^{>} \int_{0}^{1} g_{t+h-j}(s) \mathrm{d} N_{s}^{>}\right) \\
= & \mathbb{E} \int_{0}^{1} g_{t-j}(s) g_{t+h-j}(s) \mathrm{d} N_{s}^{>}+\left(\mathbb{E} N_{1}^{>}\right)^{2} \int_{0}^{1} g_{t-j}(s) \mathrm{d} s \int_{0}^{1} g_{t+h-j}(s) \mathrm{d} s \\
= & \mathbb{E} N_{1}^{>} \int_{0}^{1} g_{t-j}(s) g_{t+h-j}(s) \mathrm{d} s+\left(\mathbb{E} N_{1}^{>}\right)^{2} \int_{0}^{1} g_{t-j}(s) \mathrm{d} s \int_{0}^{1} g_{t+h-j}(s) \mathrm{d} s .
\end{aligned}
$$

Applying Bingham et al. (1989, Theorem 1.6.5), we conclude

$$
\limsup _{n \rightarrow \infty} \frac{n \mathbb{E}\left|Y_{1, n}^{>}\right|^{\delta} \mathbb{E} N_{1}^{>}}{a_{n}^{\delta}}=\limsup _{n \rightarrow \infty}\left(-\frac{n \int_{x>a_{n}}|x|^{\delta} \bar{\nu}(\mathrm{d} x)}{a_{n}^{\delta}}\right)<\infty .
$$

It holds by the Cauchy-Schwarz inequality since $f \in L^{\delta}(\mathbb{R}), \delta=2 \eta<\alpha, \delta \leq 1$, that

$$
\begin{aligned}
& \frac{1}{n} \sum_{t=1}^{n} \sum_{\substack{|j-t-h|>m \\
j=t+h+m}} \int_{j-t}^{j-t+1}|f(-s) f(h-s)|^{\eta} \mathrm{d} s=\int_{|s-h|>m}|f(-s) f(h-s)|^{\eta} \mathrm{d} s \\
& \leq\left(\int_{|s-h|>m}|f(-s)|^{\delta} \mathrm{d} s \int_{|s-h|>m}|f(h-s)|^{\delta} \mathrm{d} s\right)^{1 / 2} \rightarrow 0 \quad(m \rightarrow \infty) .
\end{aligned}
$$


Similarly, applying the inequality $a b \leq a^{2}+b^{2}$ for $a, b \geq 0$ and again the CauchySchwarz inequality we conclude

$$
\begin{aligned}
& \frac{1}{n} \sum_{t=1}^{n} \sum_{\substack{|j-t-h|>m \\
j=t+h+m}} \int_{j-t}^{j-t+1}|f(-s)|^{\eta} \mathrm{d} s \int_{j-t}^{j-t+1}|f(h-s)|^{\eta} \mathrm{d} s \\
\leq & \frac{1}{n} \sum_{t=1}^{n} \sum_{\substack{|j-t-h|>m \\
j=t+h+m}}\left(\int_{j-t}^{j-t+1}|f(-s)|^{2 \eta} \mathrm{d} s+\int_{j-t}^{j-t+1}|f(h-s)|^{2 \eta} \mathrm{d} s\right) \\
= & \int_{|s-h|>m}|f(-s)|^{2 \eta} \mathrm{d} s+\int_{|s-h|>m}|f(h-s)|^{2 \eta} \mathrm{d} s \rightarrow 0 \quad(m \rightarrow \infty) .
\end{aligned}
$$

Hence by equations (4.35)-(4.39) and since $\mathbb{E} N_{1}^{>}=\nu\left(|x|>a_{n}\right)<C$ for some $C>0$ and all $n \in \mathbb{N}, \lim _{m \rightarrow \infty} \lim \sup _{n \rightarrow \infty} a_{n}^{-2 \eta} \sum_{t=1}^{n} \sum_{\substack{|j-t-h|>m \\ j=t+h+m}}, \mathbb{E}\left|Z_{t, j}^{>} Z_{t+h, j}^{>}\right|^{\eta}=0$. Next, we consider the second term in (4.34). Analogously to the calculations before, we bound

$$
\mathbb{E}\left|Z_{t, k}^{>}\right|^{2 \eta}=\mathbb{E}\left|\int_{0}^{1} f(t-k-s) \mathrm{d} L_{s}^{>}\right|^{2 \eta} \leq \mathbb{E}\left|Y_{1, n}^{>}\right|^{2 \eta} \mathbb{E} N_{1}^{>} \int_{k-t}^{k-t+1}|f(-s)|^{2 \eta} \mathrm{d} s .
$$

Hence the latter estimation yields for $d_{k}=\int_{k}^{k+1}|f(-s)|^{2 \eta} \mathrm{d} s$ and for the second term in (4.34)

$$
\begin{aligned}
& \frac{1}{a_{n}^{4 \eta}} \sum_{t=1}^{n} \sum_{\substack{j-t-h \mid>m, j=t+h+m}} \sum_{k \in \mathbb{Z}, k \neq j} \mathbb{E}\left|Z_{t, k}^{>}\right|^{2 \eta} \mathbb{E}\left|Z_{t+h, j}^{>}\right|^{2 \eta} \\
& \leq\left(\frac{n \mathbb{E}\left|Y_{1, n}\right|^{2 \eta} \mathbb{E} N_{1}^{>}}{a_{n}^{2 \eta}}\right)^{2} \frac{1}{n} \sum_{t=1}^{n} \sum_{\substack{j-t-h \mid>m, j=t+h+m}} \sum_{k \in \mathbb{Z}, k \neq j} d_{k-t} d_{j-t-h},
\end{aligned}
$$

and as $m \rightarrow \infty$

$$
\begin{aligned}
\frac{1}{n} \sum_{t=1}^{n} \sum_{\substack{j-t-h \mid>m, j=t+h+m}} \sum_{\substack{k \in \mathbb{Z} \\
k \neq j}} d_{k-t} d_{j-t-h} & =\sum_{\substack{|j-h|>m, k \in \mathbb{Z}, j=m+h}} \sum_{\substack{k \neq j \\
k}} d_{k} d_{j-h} \\
& \leq \int_{\mathbb{R}}|f(-s)|^{2 \eta} \mathrm{d} s \int_{|s|>m}|f(-s)|^{2 \eta} \mathrm{d} s \rightarrow 0 .
\end{aligned}
$$

Hence the $\lim _{m \rightarrow \infty} \limsup \operatorname{su}_{n \rightarrow \infty}$ in (4.34) is zero by (4.37) and (4.40)-(4.41). Next we consider the first term in (4.33). By Chebyshev's inequality,

$$
\begin{aligned}
& \mathbb{P}\left(a_{n}^{-2}\left|\sum_{t=1}^{n} \sum_{k \in \mathbb{Z}} \sum_{\substack{j-t-h \mid>m \\
j=t+h+m}} Z_{t, k}^{\leq} Z_{t+h, j}^{\leq}\right|>\frac{\epsilon}{8}\right) \\
& \quad \leq \frac{8}{\epsilon a_{n}^{2}} \sum_{t=1}^{n}\left(\sum_{\substack{|j-t-h|>m, j=t+h+m}} \mathbb{E}\left|Z_{t, j}^{\leq} Z_{t+h, j}^{\leq}\right|+\sum_{\substack{|j-t-h|>m, j=t+h+m}} \sum_{k \in \mathbb{Z}, k \neq j} \mathbb{E}\left|Z_{t, k}^{\leq} Z_{t+h, j}^{\leq}\right|\right) .
\end{aligned}
$$


Since $f$ is bounded, applying the compound Poisson representation from (L2) and the Cauchy-Schwarz inequality yields for $e_{k}=\int_{k}^{k+1}|f(-s)| \mathrm{d} s$ and some $C>0$

$$
\begin{aligned}
& \mathbb{E}\left|Z_{t, j}^{\leq} Z_{t+h, j}^{\leq}\right| \\
\leq & \mathbb{E}\left(|\gamma| e_{j-t}+\sum_{i=1}^{N_{1}^{\leq}}\left|f\left(t-j-T_{i}^{\leq}\right) Y_{i, n}^{\leq}\right|\right)\left(|\gamma| e_{j-t-h}+\sum_{l=1}^{N_{1}^{\leq}}\left|f\left(t+h-j-T_{l}^{\leq}\right) Y_{l, n}^{\leq}\right|\right) \\
\leq & C\left(e_{j-t}+e_{j-t-h}\right)\left(1+2 \mathbb{E}\left|Y_{1, n}^{\leq}\right| \mathbb{E} N_{1}^{\leq}+\mathbb{E}\left|Y_{1, n}^{\leq}\right|^{2} \mathbb{E} N_{1}^{\leq}\right) .
\end{aligned}
$$

Similarly, for $j \neq k$

$$
\mathbb{E}\left|Z_{t, k}^{\leq}\right| \mathbb{E}\left|Z_{t+h, j}^{\leq}\right| \leq\left(|\gamma|+\mathbb{E}\left|Y_{1, n}^{\leq}\right| \mathbb{E} N_{1}^{\leq}\right)^{2} \int_{0}^{1}|f(t-k-s)| \mathrm{d} s \int_{0}^{1}|f(t+h-j-s)| \mathrm{d} s .
$$

Applying Bingham et al. (1989, Theorem 1.6.4), we conclude

$$
\limsup _{n \rightarrow \infty} \frac{n \mathbb{E}\left|Y_{1, n}^{\leq}\right|^{2} \mathbb{E} N_{1}^{\leq}}{a_{n}^{2}}=\limsup _{n \rightarrow \infty}\left(-\frac{n \int_{1<x \leq a_{n}}|x|^{2} \bar{\nu}(\mathrm{d} x)}{a_{n}^{2}}\right)<\infty .
$$

Since $\mathbb{E}\left|Y_{1, n}^{\leq}\right| \leq\left(\mathbb{E}\left|Y_{1, n}^{\leq}\right|^{2}\right)^{1 / 2}$ also $\lim _{\sup } \sin _{n \rightarrow \infty} n a_{n}^{-2} \mathbb{E}\left|Y_{1, n}^{\leq}\right| \mathbb{E} N_{1}^{\leq}<\infty$ and it holds that

$$
\limsup _{n \rightarrow \infty} \frac{n}{a_{n}^{2}}\left(|\gamma|+\mathbb{E}\left|Y_{1, n}^{\leq}\right| \mathbb{E} N_{1}^{\leq}\right)^{2}<\infty .
$$

These estimates and similar calculations as in (4.38) and (4.41) show that the $\lim _{m \rightarrow \infty} \limsup _{n \rightarrow \infty}$ of (4.42) is zero. Also by similar calculations it can be shown that the $\lim _{m \rightarrow \infty} \lim \sup _{n \rightarrow \infty}$ of the second and fourth term in (4.33) is zero. Next we consider the 5 th term in (4.33). By Chebyshev's inequality,

$$
\begin{aligned}
\mathbb{P}\left(\left|\sum_{t=1}^{n} \sum_{k \in \mathbb{Z}} \sum_{\substack{j-t-h \mid>m, j=t+h+m}} Z_{t, k}^{>} Z_{t+h, j}^{\leq}\right|\right. & \left.>\frac{\epsilon a_{n}^{2}}{8}\right) \\
& \leq \frac{8^{\delta}}{\epsilon^{\delta} a_{n}^{2 \delta}} \sum_{t=1}^{n} \sum_{\substack{k \in \mathbb{Z} \\
|j-t-h|>m, j=t+h+m}} \mathbb{E}\left|Z_{t, k}^{>}\right|^{\delta} \mathbb{E}\left|Z_{t+h, j}^{\leq}\right|^{\delta}
\end{aligned}
$$

Since $\sup _{n \in \mathbb{N}} \mathbb{E}\left|Y_{1, n}^{\leq}\right|^{\delta} \leq \mathbb{E}\left|Y_{1}^{+}\right|^{\delta}<\infty$ we conclude for some $C>0$

$$
\mathbb{E}\left|Z_{t, k}^{\leq}\right|^{\delta} \leq\left(|\gamma|^{\delta}+\mathbb{E}\left|Y_{1, n}^{\leq}\right|^{\delta} \mathbb{E} N_{1}^{\leq}\right) \int_{k-t}^{k-t+1}|f(-s)|^{\delta} \mathrm{d} s \leq C \int_{k-t}^{k-t+1}|f(-s)|^{\delta} \mathrm{d} s
$$

and as shown before it holds that $\mathbb{E}\left|Z_{t, k}^{>}\right|^{\delta} \leq \mathbb{E}\left|Y_{1, n}^{>}\right|^{\delta} \mathbb{E} N_{1}^{>} \int_{k-t}^{k-t+1}|f(-s)|^{\delta} \mathrm{d} s$. Hence

$$
\begin{aligned}
& \frac{1}{a_{n}^{2 \delta}} \sum_{t=1}^{n} \sum_{\substack{k \in \mathbb{Z} \\
|j-t-h|>m, j=t+h+m}} \mathbb{E}\left|Z_{t, k}^{>}\right|^{\delta} \mathbb{E}\left|Z_{t+h, j}^{\leq}\right|^{\delta} \\
& \leq \frac{C \mathbb{E}\left|Y_{1, n}^{>}\right|^{\delta} \mathbb{E} N_{1}^{>}}{a_{n}^{2 \delta}} \sum_{t=1}^{n} \sum_{\substack{k \in \mathbb{Z} \\
|j-t-h|>m \\
j=t+h+m}} \sum_{k-t}^{k-t+1}|f(-s)|^{\delta} \mathrm{d} s \int_{j-t-h}^{j-t-h+1}|f(-s)|^{\delta} \mathrm{d} s .
\end{aligned}
$$

By (4.37) and (4.41) it follows now that the $\lim _{m \rightarrow \infty} \lim \sup _{n \rightarrow \infty}$ of (4.44) is equal to zero. The 6th to 8th terms in (4.33) can be estimated analogously and therefore we proved (4.31) and the theorem in the case $c_{h, 1}^{+}+c_{h, 1}^{-}>0$. If $c_{h, 1}^{+}+c_{h, 1}^{-}=0$, there is an increasing subsequence $\left(m_{i}\right)_{i \in \mathbb{N}} \subset \mathbb{N}$, i.e. $m_{i} \leq m_{i+1}$ for all $i \in \mathbb{N}$, such that either $c_{h, 1, m_{i}}^{+}+c_{h, 1, m_{i}}^{-}=0$ for all $i \in \mathbb{N}$ or such that $c_{h, 1, m_{i}}^{+}+c_{h, 1, m_{i}}^{-}>$ 
0 for all $i \in \mathbb{N}$. In the first case $a_{n}^{-2} \sum_{t=1}^{n} X_{t, m_{i}}^{+} X_{t+h, m_{i}}^{+} \stackrel{\mathbb{P}}{\rightarrow} 0$ as $n \rightarrow \infty$ for every $i \in \mathbb{N}$ by Proposition 4 and the claim follows from (4.31), which has been already proved. Let $\delta_{0}$ denote the Dirac measure at zero. In the second case $a_{n}^{-2} \sum_{t=1}^{n} X_{t, m_{i}}^{+} X_{t+h, m_{i}}^{+} \stackrel{d}{\rightarrow} S_{\alpha / 2}\left(\beta_{h, 1, m_{i}}, \gamma_{h, 1, m_{i}}, 0\right)$ as $n \rightarrow \infty$ by Proposition 4 and $S_{\alpha / 2}\left(\beta_{h, 1, m_{i}}, \gamma_{h, 1, m_{i}}, 0\right) \stackrel{w}{\rightarrow} \delta_{0}$ for $i \rightarrow \infty$ by (3.1), since $\left|\gamma_{h, 1, m_{i}}\right|^{\alpha / 2} \rightarrow 0$ as well as $\gamma_{h, 1, m_{i}}^{\alpha / 2} \beta_{h, 1, m_{i}} \rightarrow 0$ for $i \rightarrow \infty$.

\section{Acknowledgements}

I would like to thank Alexander Lindner for fruitful discussions on this topic and I am grateful for the support by Deutsche Forschungsgemeinschaft Grant LI 1026/4-2.

\section{References}

S. Bai, M. S. Ginovyan and M. S. Taqqu. Limit theorems for quadratic forms of Lévy-driven continuous-time linear processes. Stochastic Process. Appl. 126 (4), 1036-1065 (2016). MR3461190.

K. Bartkiewicz, A. Jakubowski, T. Mikosch and O. Wintenberger. Stable limits for sums of dependent infinite variance random variables. Probab. Theory Related Fields 150 (3-4), 337-372 (2011). MR2824860.

P. Billingsley. Convergence of probability measures. John Wiley \& Sons, Inc., New York-London-Sydney (1968). MR0233396.

N. H. Bingham, C. M. Goldie and J. L. Teugels. Regular variation, volume 27 of Encyclopedia of Mathematics and its Applications. Cambridge University Press, Cambridge (1989). ISBN 0-521-37943-1. MR1015093.

P. J. Brockwell, V. Ferrazzano and C. Klüppelberg. High-frequency sampling and kernel estimation for continuous-time moving average processes. J. Time Series Anal. 34 (3), 385-404 (2013). MR3055494.

S. Cohen and A. Lindner. A central limit theorem for the sample autocorrelations of a Lévy driven continuous time moving average process. J. Statist. Plann. Inference 143 (8), 1295-1306 (2013). MR3055748.

R. Davis and S. Resnick. Limit theory for moving averages of random variables with regularly varying tail probabilities. Ann. Probab. 13 (1), 179-195 (1985). MR770636.

P. Embrechts, C. M. Goldie and N. Veraverbeke. Subexponentiality and infinite divisibility. Z. Wahrsch. Verw. Gebiete 49 (3), 335-347 (1979). MR547833.

V. Fasen. Extremes of regularly varying Lévy-driven mixed moving average processes. Adv. in Appl. Probab. 37 (4), 993-1014 (2005). MR2193993.

V. Fasen. Statistical estimation of multivariate Ornstein-Uhlenbeck processes and applications to co-integration. J. Econometrics 172 (2), 325-337 (2013). MR3010621.

V. Fasen and F. Fuchs. On the limit behavior of the periodogram of high-frequency sampled stable CARMA processes. Stochastic Process. Appl. 123 (1), 229-273 (2013). MR2988117.

I. García, C. Klüppelberg and G. Müller. Estimation of stable CARMA models with an application to electricity spot prices. Stat. Model. 11 (5), 447-470 (2011). MR2907838. 
H. Hult and F. Lindskog. On regular variation for infinitely divisible random vectors and additive processes. Adv. in Appl. Probab. 38 (1), 134-148 (2006). MR2213968.

A. Jakubowski. Minimal conditions in p-stable limit theorems. Stochastic Process. Appl. 44 (2), 291-327 (1993). MR1200412.

A. Jakubowski. Minimal conditions in p-stable limit theorems. II. Stochastic Process. Appl. 68 (1), 1-20 (1997). MR1454576.

O. Kallenberg. Random measures. Akademie-Verlag, Berlin; Academic Press, Inc. [Harcourt Brace Jovanovich, Publishers], London, third edition (1983). ISBN 0-12-394960-2. MR818219.

F. Lindskog, S. I. Resnick and J. Roy. Regularly varying measures on metric spaces: hidden regular variation and hidden jumps. Probab. Surv. 11, 270-314 (2014). MR3271332.

M. Moser and R. Stelzer. Tail behavior of multivariate Lévy-driven mixed moving average processes and supOU stochastic volatility models. Adv. in Appl. Probab. 43 (4), 1109-1135 (2011). MR2867948.

J. P. Nolan. Stable Distributions - Models for Heavy Tailed Data. Birkhäuser, Boston (2017). In progress, Chapter 1 online at http://fs2.american.edu/jpnolan/www/stable/stable.html.

A. G. Pakes. Convolution equivalence and infinite divisibility: corrections and corollaries. J. Appl. Probab. 44 (2), 295-305 (2007). MR2340199.

B. S. Rajput and J. Rosiński. Spectral representations of infinitely divisible processes. Probab. Theory Related Fields 82 (3), 451-487 (1989). MR1001524.

K. Sato. Lévy processes and infinitely divisible distributions, volume 68 of Cambridge Studies in Advanced Mathematics. Cambridge University Press, Cambridge (1999). ISBN 0-521-55302-4. MR1739520.

K. Sato. Additive processes and stochastic integrals. Illinois J. Math. 50 (1-4), 825-851 (2006). MR2247848.

F. Spangenberg. A central limit theorem for the sample autocovariance of a continuous time moving average process with long memory. ArXiv Mathematics e-prints (2015). arXiv: 1502.04851. 